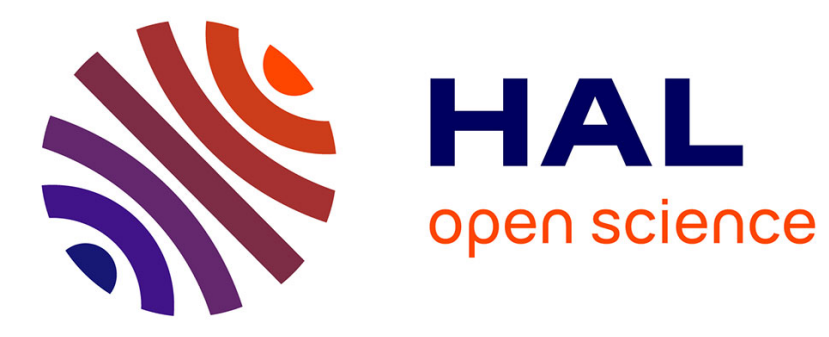

\title{
Theoretical study of the pyrolysis of $\beta-1,4-x y l a n:$ a detailed investigation on unimolecular concerted reactions
}

\author{
M. Goussougli, B. Sirjean, P.-A. Glaude, R. Fournet
}

\section{- To cite this version:}

M. Goussougli, B. Sirjean, P.-A. Glaude, R. Fournet. Theoretical study of the pyrolysis of $\beta-1,4$-xylan: a detailed investigation on unimolecular concerted reactions. Physical Chemistry Chemical Physics, 2021, 23 (4), pp.2605-2621. 10.1039/D0CP06024K . hal-03195939

\author{
HAL Id: hal-03195939 \\ https://hal.science/hal-03195939
}

Submitted on 28 Sep 2021

HAL is a multi-disciplinary open access archive for the deposit and dissemination of scientific research documents, whether they are published or not. The documents may come from teaching and research institutions in France or abroad, or from public or private research centers.
L'archive ouverte pluridisciplinaire HAL, est destinée au dépôt et à la diffusion de documents scientifiques de niveau recherche, publiés ou non, émanant des établissements d'enseignement et de recherche français ou étrangers, des laboratoires publics ou privés. 


\title{
Theoretical study of the pyrolysis of $\beta-1,4-x y l a n:$ a detailed investigation on unimolecular concerted reactions.
}

\begin{abstract}
M. Goussougli, ${ }^{a}$ B. Sirjean ${ }^{a}$, P.-A. Glaude ${ }^{a}$ and R. Fournet ${ }^{a,{ }^{*}}$
A theoretical study of the thermal decomposition of $\beta-1,4-x y l a n$, a model polymer of hemicelluloses, is proposed for the first time. A mechanism based on unimolecular concerted reactions is elaborated in a comprehensive way. Elementary reactions, such as dehydrations, retro-aldol, retro Diels-Alder, retroene, glycosidic bond fissions, isomerizations, etc., are applied to $\beta-1,4-x y l a n$, as well as to the fragments formed. At each stage of the construction of the mechanism, the fragments previously retained are decomposed and the low energy paths are selected to define new fragments. Energy barriers are computed at the CBS-QB3 level of theory and rate coefficients of important reactions are calculated. It is shown that the main reaction pathways can be modelled by reactions involving two specific fragments, which react in closed sequences, similarly to chain-propagating reactions. The proposed reaction scheme allows to predict important species observed during the pyrolysis of xylan, such as aldehydes or CO. In addition, we show that dehydrations require high activation energy and cannot compete with the other reactions. Therefore, it seems difficult to explain, by means of unimolecular homogeneous gas phase reactions, the significant formation of specific species such as furfural as reported by several authors.
\end{abstract}

\section{Introduction}

The increase in greenhouse gases, combined with a growing energy demand, requires finding alternative resources to fossil fuels. Lignocellulosic biomass is an interesting renewable raw material for the production of bio-fuels and chemicals. Many conversion processes have been developed to extract organic compounds from biomass and fast pyrolysis represents an effective way to produce bio-oils in high yields ${ }^{1}$. However, to help in the design of reactors, to reduce the cost of production and to promote the formation of high-quality products, it is necessary to better understand the chemistry involved in such transformations ${ }^{2}$.

Lignocellulosic biomass is mainly composed of cellulose, lignin and hemicellulose. If the pyrolysis of cellulose has been extensively studied, less experimental and theoretical works have been devoted to hemicellulose ${ }^{3}$. Hemicelluloses are complex polysaccharides with a skeleton containing monomers such as xylose, mannose or glucose, associated with many possible side chains such as acetyl, glucuronic, galactose or arabinose structures ${ }^{4}$. The kinetic modelling of hemicellulose pyrolysis represents a considerable challenge due to its structural complexity. Several theoretical studies based on model molecules (usually xylose or xylobiose) have been performed to elucidate the main reaction pathways. Wang et al. ${ }^{5}$ performed a DFT study of the pyrolysis of $\beta$-D-xylose at the B3LYP/6-31++G(d,p) level of theory. Several reaction pathways have been investigated to explain the formation of the products observed experimentally. The most favourable route found is a concerted reaction involving the ring opening by breaking the $\mathrm{C}-\mathrm{O}$ bond. The product can undergo two successive dehydrations, followed by a $C_{5}$ ring closure to form furfural. It can be noted that the second dehydration cannot occur in the $\beta-1,4-x y l a n$ due to the presence of a xylose group instead of a $\mathrm{H}$ atom in $\beta$-D-xylose. Huang et al. ${ }^{6,7}$ performed a theoretical kinetic study of the thermal degradation of xylose as a hemicellulose model compound. Calculations performed at the M06-2X/6-31++G(d,p) level of theory allowed the authors to propose eight reaction pathways leading to ultimate decomposition products. In accordance with the work of Wang et al., they found that the easiest pathway involves the direct ring-opening to form an acyclic carbonyl-containing isomer. The pyrolysis of this compound has been investigated and four possible routes have been proposed, which lead to the formation of products such as methylglyoxal, glycoaldehyde, furfural, acetone, acetaldehyde, and CO. The authors also mentioned two other important decomposition pathways: a direct decomposition yielding ethenol, water and 2-hydroxymalondialdehyde and a dehydration reaction leading to unsaturated cyclic species, which in turn lead to the formation of products encountered in the reactions of the acyclic carbonyl-containing isomer discussed above. Even if these previous theoretical studies of the literature permitted to highlight the mechanisms involved in the thermal degradation of a single xylose unit, they are not well adapted to model xylan or hemicellulose since glycosidic bonds are not considered. $\mathrm{Li}$ et al. ${ }^{8}$ performed a theoretical study on the mechanism of xylobiose decomposition during pyrolysis process. Potential energy profiles were obtained at the M06-2X/6-31+G(d,p) level of theory. They showed that the initiation reaction involving the ring opening is more favourable than the glycosidic bond breaking by more than $70 \mathrm{~kJ} \mathrm{~mol}^{-1}$. They also investigated the subsequent decompositions of the intermediates formed from the ring opening. Especially, retro-aldol reactions have been identified as preferred routes to aldehydes, while dehydration seems to be the major reaction channel to form formaldehyde and 1-hydroxy-2-propanone. More recently, Zhou et al. ${ }^{9}$ proposed a detailed modelling of the thermal degradation of hemicellulose (from corn stover) based on unimolecular concerted reactions. The model molecule considered is an arabinoxylan structure with a variable degree of polymerization (DP). The generic reactions as well as the rate coefficients proposed are mainly deduced by analogy with cellulose pyrolysis. The comparison between simulations and experimental results, reported by Zhang et al. ${ }^{10}$ for fast pyrolysis of extracted hemicellulose from corn stover at $500^{\circ} \mathrm{C}$, shows a good agreement with the major products observed. It is shown that the degree of polymerization (DP) does not modify strongly the product distribution for values higher than 30 but for DP around 15, substantial differences are observed for specific products such as dianhydroxylopyranose or glycoaldehyde, due to the competition between end-chains and mid-chain reactions.

Yang et al. ${ }^{11}$ studied, by TGA-FITR and fast pyrolysis experiments, the thermal degradation of hemicellulose from beech wood. Especially, they measured the relative yields of main products for temperatures ranging from 300 to $600^{\circ} \mathrm{C}$. In addition, they proposed reaction 
pathways, based on DFT calculations (M06-2X/def2tzvp//B3LYP/6-31G(d,p)), to explain the main products formed during the pyrolysis of xylose, xylobiose and 4-O-methylglucuronic acid groups. In the same way, Hu et al. ${ }^{12}$ performed an experimental and theoretical study on the fast pyrolysis of xylose, xylobiose and xylan (from birch wood). Experiments were performed by using a pyroprobe pyrolyser coupled to a GC-MS. At $400^{\circ} \mathrm{C}$, hydroxyacetaldehyde (HAA), furfural (FF), dianhydro xylose (DAX) and 1,4-anhydro-D-xylopyranose (ADX) are the main products identified from xylose and xylobiose while for xylan, the predominant products are: HAA, hydroxyacetone (HA), 1-hydroxy-2butanone (HB), and in a lesser extent, furfural. A mechanistic investigation has been performed for the xylose pyrolysis at the B3LYP/6$311 G(d, p)$ level of theory (with empirical dispersion "D3"), in order to explain the formation of the main products observed experimentally. The crucial role of D-xylose and its subsequent reaction pathways have been highlighted. In the same way, Gibbs free energies have been computed for initiation reactions involved in the thermal degradation of xylobiose and the results showed that important intermediates formed are similar to those produced in the pyrolysis of xylose. Finally, a formal discussion based on the high degree of polymerization of xylan and the role of the glycosidic bond, are proposed to explain the difference between the major products found experimentally.

Yu et al. ${ }^{13}$ investigated the reaction mechanism of fast pyrolysis of xylobiose and xylotriose by means of a fast pyrolysis probe coupled with a high-resolution mass spectrometer. They identified at least ten primary products. They proposed several reaction pathways to explain their formation, based on electronic structure calculations at the $M 062 X / 6-311++G(d, p)$ level of theory. Free energy barriers were calculated for initiation reactions such as dehydrations, glycosidic bond breaking and ring contraction. This study confirmed that the fragmentation of the chain end ("reducing end-chain") via ring-opening and retro-aldol reactions is the dominant pathway for xylobiose decomposition. In addition, it is proposed that the dissociation of glycosidic bonds or the loss of water can occur via ring contraction or direct dehydration respectively.

The previous theoretical studies focused on the thermal decomposition of the xylose part of xylan or, to a lesser extent, the breaking of the glycosidic bond in xylobiose. However, a detailed and comprehensive mechanism including all the decomposition steps of $\beta-1,4-x y l a n$ has never been investigated. The presence of polycycles, linked by glycosidic bonds, involves new fragments and specific reaction pathways. In the present work, we performed a systematic investigation of the mechanism of the pyrolysis of $\beta-1,4-x y l a n$, by means of electronic structure calculations performed at the CBS-QB3 level of theory. This composite method is more reliable to compute energies than DFT methods such as B3LYP ${ }^{14,15}$. The different decomposition pathways investigated involved unimolecular concerted reactions and have been explored in a comprehensive way. At each step, the main reaction pathways have been selected and the fragments obtained have been decomposed by considering the lowest energy paths. Rate coefficients were computed for all important reactions. Comparisons with literature data are made, when possible, to identify products observed experimentally or from ab initio calculations performed on model molecules such as xylose or xylobiose. Finally, based on the previous results, we propose a detailed mechanism of the thermal decomposition of $\beta-1,4-x y l a n$ and we show that it is possible to introduce close sequence reactions similar to chain-propagating reactions, involving two specific chemical structures.

\section{Methodology and computational details.}

All the potential energy profiles have been computed using the CBS-QB3 method implemented in Gaussian 09 Rev. D.01 ${ }^{16}$. It involves geometry optimizations and frequency calculations at the B3LYP/6-311G(d,p) level of theory. However, in order to better describe hydrogen bonds, we added diffuse functions for all atoms (B3LYP/6-311++G(d,p)). In this study, a comparison of the barrier heights involved in radical and concerted reactions have been performed for initiations reactions. To correctly described diradicals formed during the ring opening of xylan, we used a modified version of CBS-QB3, presented in ref. ${ }^{17}$. This method allows to deduce the energy of open

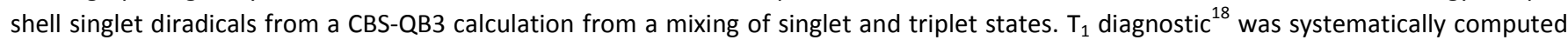
to check the quality of single-reference electron correlation methods. We found that $T_{1}$ is lower than 0.02 for singlet states, which is consistent with the use of a mono-determinantal method. Electronic energy derived from a CBS-QB3 calculation is obtained from three levels of theory (including a $\operatorname{CCSD}(\mathrm{T})$ method) coupled to three different basis set to reach the complete basis set (CBS) and to obtain a good correlation energy. As the $\operatorname{CCSD}(\mathrm{T})$ calculation is time consuming, we systematically construct a model molecule for each elementary reaction, in order to correctly describe, with the minimum of heavy atoms, the reactional moiety. This number can be substantial, especially for initiation reactions (up to 18 heavy atoms). In most cases, model molecules contain two cycles linked by a glycosidic bond. When a reaction involves a lateral chain on the cycle, and that no hydrogen bond is engaged during the reaction with an adjacent cycle, only one xylose monomer has been considered to describe the model molecule. In the same way, only hydroxyl groups interacting in hydrogen bonds with the reaction moiety, have been kept in the model molecule". Cartesian coordinates of the model molecules used in our calculations are given in the ESI. The lower energy conformer was selected by performing internal rotations (relaxed scans) around the $\mathrm{C}-\mathrm{OH}$ bonds. Intrinsic Reaction Coordinate (IRC) has been performed to check that transition states (TS) correctly connect the reactants and the products when complex reaction mechanisms were involved (ring contraction, ring closing/opening, etc.). Rate constants have been computed based on canonical transition state theory coupled with statistical thermodynamics to evaluate the partition functions (equation 1):

$$
k_{\infty}(T)=\kappa L \frac{k_{B} T}{h} \frac{Q_{T S}(T)}{Q_{R}(T)} \exp \left[-\frac{V^{\ddagger}}{R T}\right]
$$


where $k_{B}$ is Boltzmann's constant, $h$ is Planck's constant, $T$ the temperature and $V^{\neq}$, the classical barrier height. $Q_{T S}(T)$ and $Q_{R}(T)$ represent the partition function calculated, respectively, for the transition state (TS) and the reactant (R). $L$ is the statistical factor and take into account external symmetries and optical isomers of the reactants and the TS. $\kappa$ is the transmission coefficient and it is computed from a one dimensional asymmetric Eckart potential ${ }^{19}$. If vibrational partition functions have been calculated based on harmonic oscillator $(\mathrm{OH})$, vibrations corresponding to internal rotation were replaced by hindered rotors from a modified 1D-HR approach described in ref ${ }^{20}$. This approach was applied for internal rotations of hydroxyl groups, acyclic parts of fragments and chain ends. Internal rotations around the glycosidic bonds between two xylose groups were considered as sufficiently well described by a harmonic oscillator, due to the constraint induced by the high degree of polymerization of $\beta$ 1,4-xylan.

Finally, we fitted rate coefficients from equation (1) over the temperatures range 500-1200 K, from a three parameter Arrhenius expression (equation 2):

$$
k_{\infty}(T)=A T^{n} e^{-\frac{E}{R T}}
$$

\section{Results and discussion.}

\section{Conformational analysis of $\beta-1,4-x y l a n$}

As mentioned in the introduction, theoretical studies on the pyrolysis of hemicellulose have been already performed on simple model molecules, such as xylose or xylobiose. $\beta-1,4-x y l a n$ has a backbone composed of xylose monomers linked by glycosidic bonds and it is necessary to define the lower energy conformer prior to start potential energy surface calculations. Due to the large number of hydrogen bonds involved in such structures ${ }^{21,22}$, it is necessary to envisage several conformations related to the different $\mathrm{OH}$ orientations ${ }^{23}$. We performed several energy calculations by varying the orientation of the hydroxyl groups relative to the oxygen atoms of the glycosidic bond and the ring oxygen. Scheme 1 shows four conformations involving four xylose groups, to model the $\beta-1,4-x y l a n$. This is a minimal structure to perform a conformational analysis. Due to the high number of heavy atoms, calculations have been performed using the following dual level: b3lyp/6-311++G(d,p)//b3lyp/6-31+G(d,p). If the absolute energy computed at this level of theory is not of high accuracy, the energy difference between two structures is deemed sufficiently accurate to identify the lowest energy structure. Indeed, recent calculations performed on similar structures showed that the lowest energy conformers obtained at the B3LYP level of theory match with the lowest energy conformers obtained from CBS-QB3 calculations ${ }^{24}$.

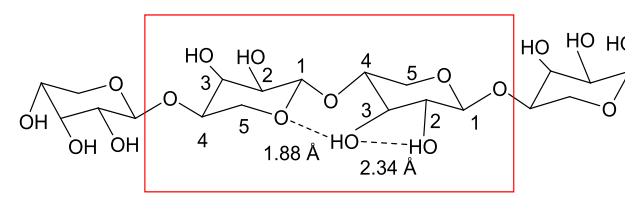

$$
\begin{aligned}
& \left(\mathrm{E}_{\mathrm{OK}, \mathrm{rel}}=0\right) \\
& \left(\mathrm{E}_{\mathrm{OK}, \mathrm{rel}}=26.8\right)
\end{aligned}
$$

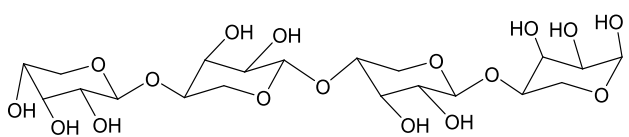

(b)
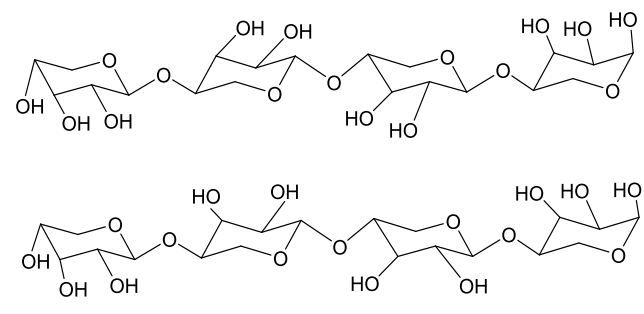

$\left(\mathrm{E}_{\mathrm{OK}, \mathrm{rel}}=13.1\right)$

(d)

Scheme.1 Relative energy at OK (including ZPE) of four conformations of model molecules of $\beta-1,4-x y l a n$, obtained at the b3lyp/6-311++G(d,p)//b3lyp/31+G(d,p) dual level. Relative energies are given in $\mathrm{kJ} \mathrm{mol}^{-1}$.

The two xylose groups outside the red square in scheme 1 represent the chain ends of $\beta-1,4-x y l a n$, while the two groups inside can be related with the mid-chains. To find the lower energy conformation, it is necessary to maximize the number of hydrogen bonds in the molecular structure. For chain end groups, it corresponds to an orientation of the hydroxyl functions as depicted in 
Scheme 1. In this conformation, all the $\mathrm{OH}$ groups are involved in a hydrogen bond. For the mid chains, several conformations can be considered. In Scheme 1.a, the hydroxyl group connected to the carbon atom \#3 gives rise to a hydrogen bond with the ring oxygen of the adjacent xylose group. The length between the hydrogen atom and the ring oxygen is equal to $1.88 \AA$, which strongly stabilizes the molecular structure. In this conformation, the hydroxyl group on the carbon \#2 is oriented towards the adjacent - $\mathrm{OH}$ (Scheme 1a) with a hydrogen bond length around $2.34 \AA$ A. Another possibility is an orientation of the hydroxyl group connected to the carbon \#2, towards the oxygen atom of the glycosidic bond and the other -OH in the same direction (Scheme 1b). However, the structure obtained is less stable, which can be explained by weaker hydrogen bond energies (hydrogen bond lengths are equal to 2.57 and $2.43 \AA$ respectively). Scheme $1 \mathrm{c}$ and Scheme $1 \mathrm{~d}$ show two other conformations constructed by mixing the $\mathrm{OH}$ orientations with respect to those previously investigated. They all lead to higher energies than that obtained in Scheme 1a, which represents the lower energy conformation found. Thus, to model the thermal degradation of mid-chains of $\beta$ 1,4-xylan from model molecules, it is necessary to consider $\mathrm{OH}$ orientations as indicated in the red square of Scheme $1 \mathrm{a}$. This point is particularly important because a correct description of the rupture and the formation of hydrogen bonds during the reaction is mandatory to avoid errors in the computed energy barriers. In the model molecules used in this study, OH group orientations were systematically kept as in Scheme 1a. In some cases, to reduce computational time, $\mathrm{OH}$ groups far from the reactive moiety were replaced by $\mathrm{H}$-atoms. In these cases, $\mathrm{OH}$ group orientation was also carefully kept. We also adapted the $\mathrm{OH}$ group orientation when fragments are formed, to take into account possible changes in hydrogen bond lengths and to capture the lower energy conformation. For fragments having an acyclic part, we systematically searched the lowest energy conformer by scanning internal rotations.

\section{Primary decomposition of the mid-chains of $\beta-1,4-x y l a n$}

We first investigated the mid-chains reactions involved in the thermal decomposition of $\beta-1,4-x y l a n$. Several elementary reactions have been proposed in the literature for the pyrolysis of $\beta-1,4-x y l a n$, using xylobiose as a model molecule ${ }^{5,6,8,9,11-13}$.

Scheme 2 shows that the unimolecular decompositions related to the cleavage of the glycosidic bond involve energy barriers ranging from 280 to $310 \mathrm{~kJ} \mathrm{~mol}^{-1}$. It is worth noting that such fragments were detected by Yu et al. ${ }^{13}$ during the decomposition of xylobiose and xylotriose. The experiments were conducted in a commercial fast pyrolysis probe coupled with a high-resolution mass spectrometer. However, the authors could not distinguish between the isomers showed in Scheme 2.

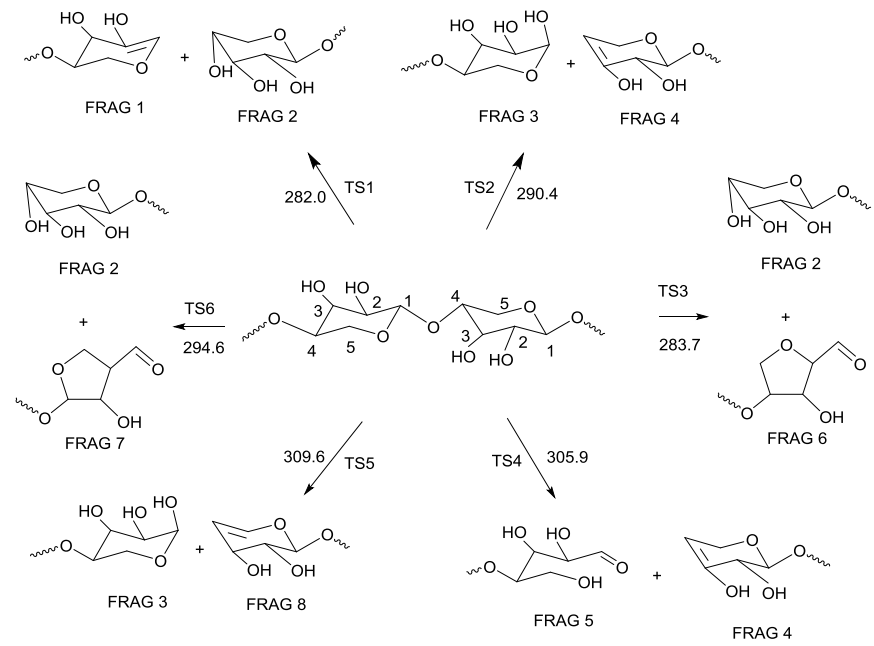

Scheme 2: Depolymerisation through concerted reactions involved in the $\beta-1,4-x y l a n$ pyrolysis. Energy barriers (at $0 \mathrm{~K}$ and including $\mathrm{ZPE}$ are given in $\mathrm{kJ}$ mol ${ }^{-1}$, at the CBS-QB3 level of theory.

Reactions through TS1, TS2 and TS5 involve a 4-centered concerted reaction (Scheme 3). The presence of a hydroxyl group on the carbon atoms 2 and 3 weakens the $\mathrm{C}-\mathrm{H}$ bond and minimizes the activation barriers of TS1 and TS2, compared to TS5. These three reactions all lead to xylose-like fragments (FRAG 2 and 3 in Scheme 2) and to unsaturated chain ends.

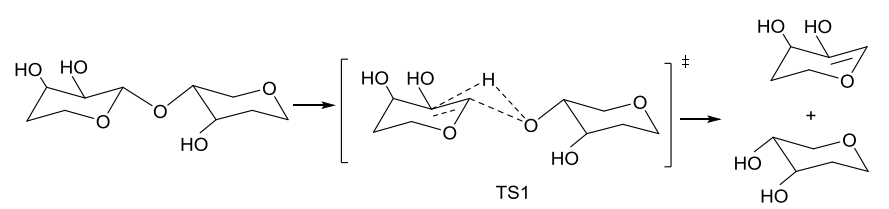

Scheme 3. TS1 involved in the 4-centered transition reaction of Scheme 2, with the model molecule used to perform the CBS-QB3 calculation. 
The xylose-like fragments are similar to the chain ends of $\beta-1,4-x y l a n$ (Scheme 1 ). Another glycosidic bond fission involves a 6 centered concerted reaction (TS4 of Scheme 2). This process yields an unsaturated end-chain and an acyclic fragment with a barrier height close to $305 \mathrm{~kJ} \mathrm{~mol}^{-1}$ (Scheme 4):

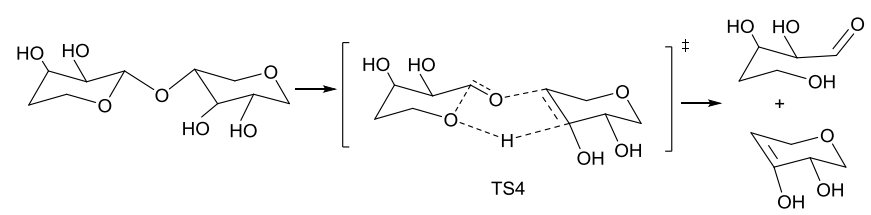

Scheme 4. TS4 involved in the 6-membered concerted reaction of Scheme 2, with the model molecule used to perform the CBS-QB3 calculation.

Even if this reaction involves a 6-centered cyclic transition state, geometric constraints due to the ring opening, enhanced by the complete rupture of the strong stabilizing hydrogen bond (between the $\mathrm{OH}$ located on carbon \#3 and the ring oxygen), leads to an increase of the energy barrier compared to TS1 or TS2.

The last glycosidic bond cleavage investigated involves a ring contraction as mentioned in ref ${ }^{12,13}$. These reactions (TS3 and TS6) involve a complex bicyclic transition state (Scheme 5).

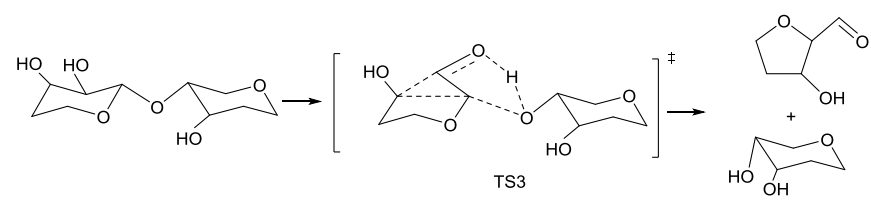

Scheme 5. TS3 involved in the glycosidic bond fission of $\beta-1,4-x y l a n$ and the $C_{5}$ ring formation (Scheme 2 ), with the model molecule used to perform the CBS-QB3 calculation.

IRC calculation confirms that the transition state correctly connects the reactant and the products. Moreover, the T1 diagnostic is equal to 0.018 , which is consistent with the mono-determinantal approach used in this work. The energy barriers involved in TS3 and TS6 are close to that computed, respectively, for TS1 and TS2.

Previous calculations in the literature have been done on xylobiose, at a lower level of theory (B3LYP) that usually underestimates barrier heights for these systems. It does not make much sense to directly compare these values with our highlevel CBS-QB3 calculations. However, Li et al $^{8}$ computed energy barriers involved in the pyrolysis of xylobiose, using the M06-2X functional. This pure DFT level of theory can lead to energy barriers closer to the composite CBS-QB3 values than B3LYP. For TS1 and TS2, Li et al. computed energy barriers equal to 277.0 and $288.3 \mathrm{~kJ} \mathrm{~mol}^{-1}$ respectively, in good agreement with our values. However, for TS5, a larger difference $\left(15.5 \mathrm{~kJ} \mathrm{~mol}^{-1}\right)$ is observed.

Among all the reactions investigated in the literature, the dehydration is often cited as an important pathway involved in the pyrolysis of hemicellulose ${ }^{9}$. Scheme 6 depicts possible dehydrations for the mid-chain patterns of $\beta-1,4-x y l a n$, with their corresponding energy barriers, computed at the CBS-QB3 level of theory. It can be seen that the dehydration reactions require higher activation energies than those involved in the cleavage of the glycosidic bond, especially when compared to TS1 and TS2. Note that the barrier height given by Li et al. ${ }^{8}$ for TS8 is equal to $326.8 \mathrm{~kJ} \mathrm{~mol}^{-1}$, in agreement with our value of $331.0 \mathrm{~kJ} \mathrm{~mol}^{-1}$.

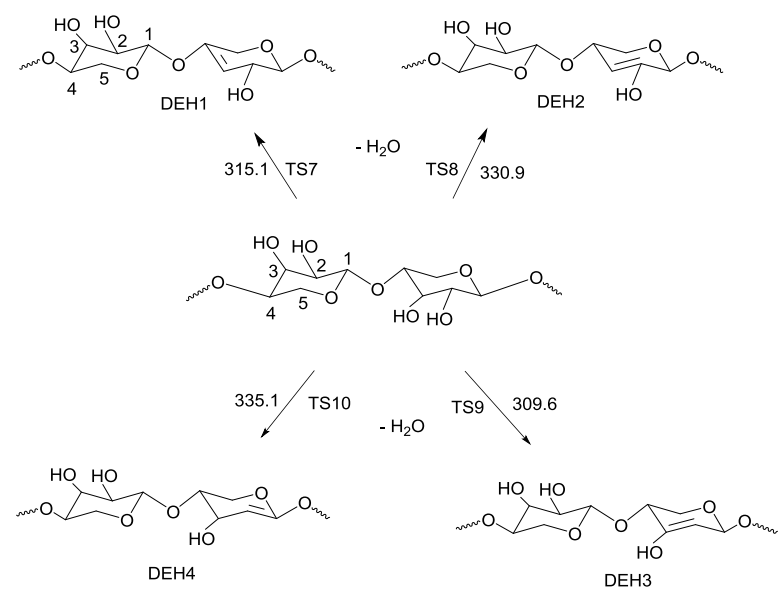


It is commonly accepted that radical processes are negligible compared to concerted reactions, during the pyrolysis of cellulose or hemicellulose ${ }^{9,25-27}$. To support this statement, we calculated the bond dissociation energies (BDE) of $\beta-1,4-x y l a n$. We also computed energy barriers involved in the formation of diradicals by homolytic ring opening, following the methodology described in the $\$ 2$. Scheme 7 summarizes calculated BDEs and corroborates the assumption that radical initiations involve high activation energies.

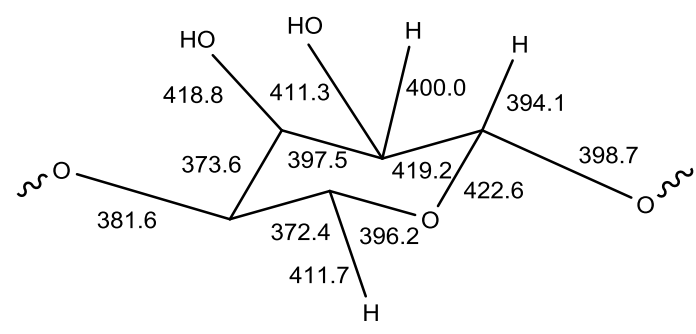

Scheme 7: Bond dissociation energies (in kJ mol ${ }^{-1}$ ) computed at the CBS-QB3 level. Values for C-C and ring C-O bonds are energy barriers.

Indeed, the lowest energy barrier found is the ring opening resulting from the cleavage of the $\mathrm{C}_{4}-\mathrm{C}_{5}$ bond $\left(372.4 \mathrm{~kJ}\right.$ mol $\left.{ }^{-1}\right)$. This energy is $90.4 \mathrm{~kJ} \mathrm{~mol}^{-1}$ higher than the lowest energy barrier involved in the concerted glycosidic bond fission.

In order to highlight the easiest decomposition pathways, we computed the rate constants, using the procedure described in section 2. Values of the rate constants for glycosidic bond breaking and dehydration reactions are depicted in Fig.1.a and 1.b, respectively, for temperatures ranging from 500 to $1000 \mathrm{~K}$, while the corresponding rate coefficients are given in Table S1.1 and Table S1.2 in the supplementary information. For comparison, the two unimolecular initiations producing radicals and having the lowest energy barriers were added (Fig.1.C). The first reaction relates to the scission of the glycosidic bond between carbon \#4 and the oxygen atom (Scheme 7), while the second reaction involves the ring opening, by breaking the $C_{4}-C_{5}$ bond. The corresponding rate coefficients are given in Table S1.3. For the first reaction, we estimated the activation energy based on the bond dissociation energy of xylobiose, at 298K. The pre-exponential factor has been computed based on the equilibrium constant and the reverse rate constant (combination), for which the A factor was taken equal to $5.010^{12} \mathrm{~cm}^{3} \mathrm{~mol}^{-1} \mathrm{~s}^{-1}$. This value is consistent with similar combination reactions involving polycycles ${ }^{28}$.

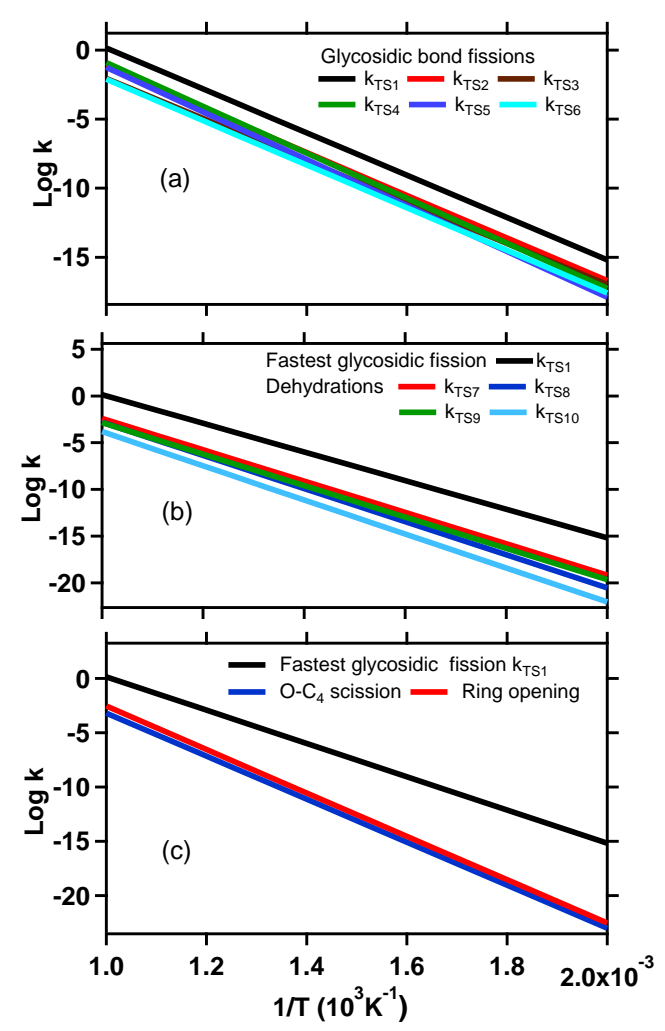

Fig. 1. Rate constants $\left(\mathrm{s}^{-1}\right)$ involved in Scheme 2, versus the inverse temperature for T ranging from 500 to $1000 \mathrm{~K}$.

Fig.1a shows that the easiest decomposition pathway of the mid-chain patterns involves a glycosidic bond fission (TS1) and leads to an unsaturated fragment (FRAG1) and a xylose-like fragment (FRAG2). At $750 \mathrm{~K}$, the ratio between the corresponding rate constant and that involving the second most important reaction (TS4) is equal to 26 . Even if the energy difference between the 
two structures $\left(23.8 \mathrm{~kJ} \mathrm{~mol}^{-1}\right.$ ) can explain the observed ratio, this is not the case for TS3 and TS2 (to a lesser extent), which have close energy barriers. For these two latter transition states, entropic effects reduce the corresponding rate constants. Indeed, in TS2 and TS3 a strong hydrogen bond (1.87 and $1.67 \AA$ A respectively) stabilizes their structure, which lower activation energies but significantly decreases their entropy. An opposite effect is observed in TS1, since the strong hydrogen bond in the reactant (1.9 $\AA$ ) is broken during the formation of the transition state. Accordingly, the pre-exponential factor in the rate constant of the first glycosidic bond fission (TS1) is well above that corresponding to TS2 and TS3.

As expected, Figure $1 \mathrm{~b}$ shows that dehydration reactions are more difficult than glycosidic bond fissions. In addition at $750 \mathrm{~K}$, a ratio of 5.1 is found between the rate constants, for the most difficult glycosidic bond fission and the easiest dehydration reaction. In the same way, radical reactions remain negligible in the temperature range considered.

\section{Primary decomposition of the chain ends of $\beta-1,4-x y l a n$}

$\beta-1,4$-xylan can also reacts at the chain ends. Chain ends can be represented by fragments 2 and 3 (Scheme2). Previous studies performed on cellulose and hemicellulose ${ }^{6,8,12,13,29}$ showed that the chain ends react differently, according to their structure (reducing end-chain (RE) and non-reducing end-chain (NR)).

- Reactions of the fragment 3

This fragment corresponds to a reducing end-chain. The hydrogen atom of the hydroxyl group bonded to the carbon \#1, can be transferred to the O-ring, leading to the ring opening (TS11 in Scheme 8). It is worth noting that this product is the fragment 5, already encountered in the mid-chain decomposition. This reaction involves a low energy barrier of $193.7 \mathrm{~kJ} \mathrm{~mol}^{-1}$, in correct agreement with the value obtained by Huang et al. ${ }^{6}\left(189.1 \mathrm{~kJ} \mathrm{~mol}^{-1}\right)$ at the M062X level of theory.

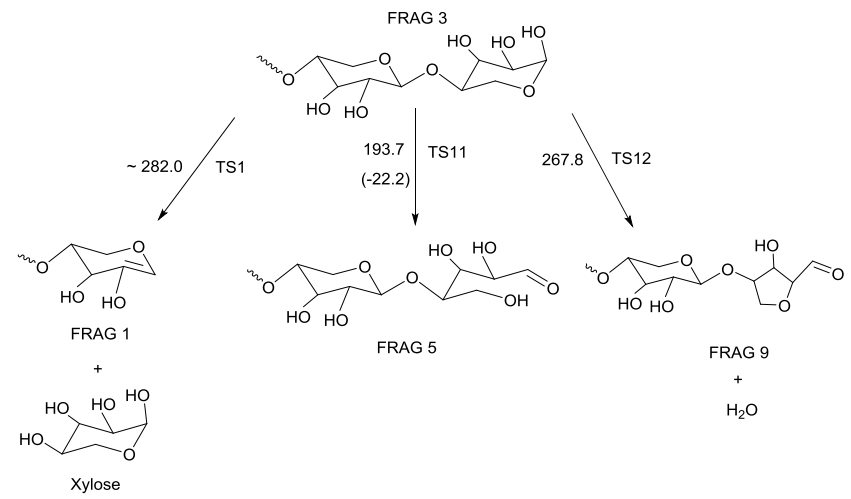

Scheme 8. Main reactions at the reducing end-chain (fragment 3 ), with the barrier heights computed at the CBS-QB3 level of theory (at $0 \mathrm{~K}$, in $\mathrm{kJ}$ mol ${ }^{-1}$ ). The value in parenthesis corresponds to the reaction enthalpy at $0 \mathrm{~K}$.

Another possible reaction is the ring contraction of the end-chain (TS12 of Scheme 8), according to the following pathway (Scheme 9):

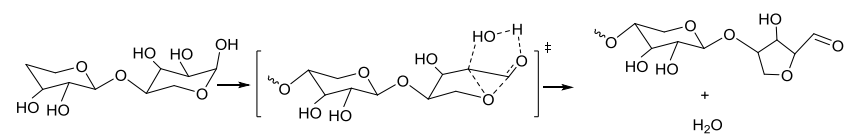

Scheme 9. Ring contraction of fragment 3 with the model molecule used to perform the CBS-QB3 calculation.

This concerted reaction involves a water elimination, combined with the formation of a tetrahydrofuran-carbaldehyde structure by a ring contraction of the end-chain. The barrier height is largely higher $\left(267.8 \mathrm{~kJ} \mathrm{~mol}^{-1}\right)$ than the ring opening. Three other similar reactions can be considered depending on the $\mathrm{H}$-atom transferred and the hydroxyl group removed. However, Yu et al. ${ }^{13}$ showed that these other reactions are less favourable than that presented in Scheme $9\left(28.0 \mathrm{~kJ} \mathrm{~mol}^{-1}\right.$ higher at least) and we did not consider their formation in this study. On the other hand, all the reactions previously described for the mid-chains can also occur in fragment 3. The previous calculations showed that the concerted reaction involving TS1 (Scheme 2) is the easiest pathway, with a barrier height of $282.0 \mathrm{~kJ} \mathrm{~mol}^{-1}$. This reaction leads to the formation of xylose and a structure similar to fragment 1. Thus, we added this reaction to Scheme 8 . The rate constants are depicted in Fig. 2, while the corresponding rate coefficients are given in Table S1.5. 


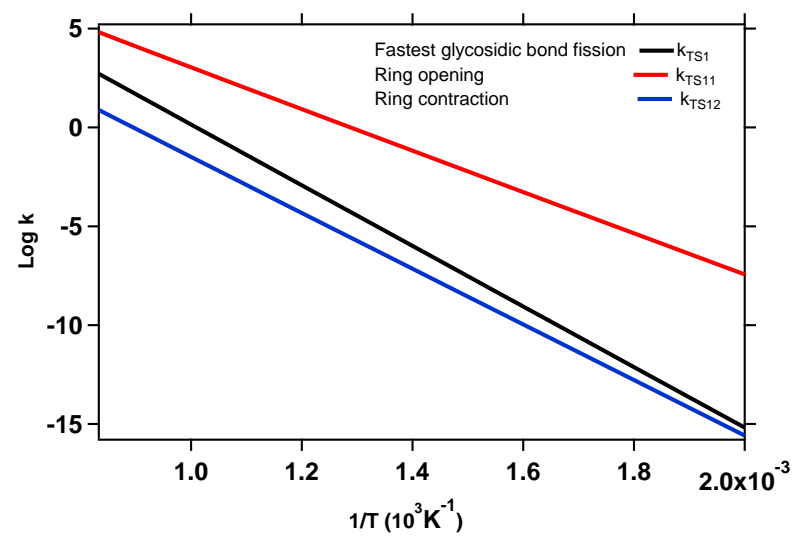

Fig. 2. Rate constants $\left(\mathrm{s}^{-1}\right)$ involved in Scheme 8 , versus the inverse temperature for T ranging from 500 to $1200 \mathrm{~K}$.

As expected, the reaction leading to the ring opening represents the dominant pathway for fragment 3 . At $750 \mathrm{~K}$, the rate constant of the formation of FRAG5 is $3.10^{4}$ times higher than the easiest glycosidic bond fission (TS1). Moreover, this reaction is slightly exothermic with a reaction enthalpy, at $0 \mathrm{~K}$, equals to $-22.2 \mathrm{~kJ} \mathrm{~mol}^{-1}$. Even if the degree of polymerization of $\beta-1,4-x y l a n$ can be large (around 200), the ring opening at the reducing end-chain seems to be the most favourable reaction pathway involved in the primary decomposition of $\beta-1,4-x y l a n$.

- Reactions of the fragment 2

This fragment corresponds to a non-reducing end-chain (NR) and cannot easily react by ring opening. Thus, the reactions involve in this structure are similar to those encountered for the mid-chains (Scheme 2). Accordingly, the most likely reaction is the glycosidic bond fission corresponding to TS1 (Scheme 10). We see that the reaction can be described by an iterative generic process, leading to the formation of an unsaturated cyclic $\mathrm{C}_{6}$ species (3,4-dihydro-2 $\mathrm{H}$-pyran-3,4,5-triol) and a fragment similar to fragment 2, but containing one less monomer.

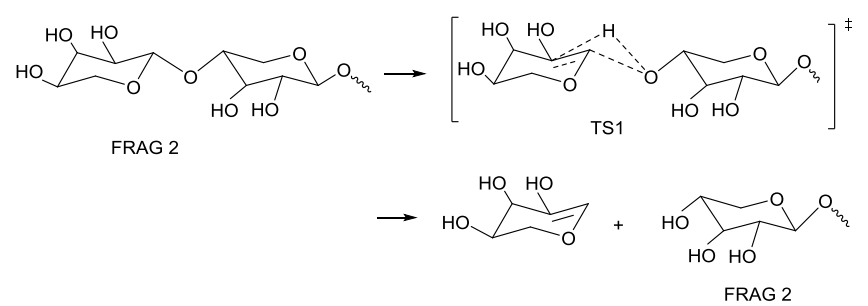

Scheme 10. Main reaction in the pyrolysis of fragment2.

Since the transition state is very close to that involves in the glycosidic bond fission of $\beta-1,4-x y l a n$ (TS1 in Scheme 2 ), it is reasonable to use the same rate constant for this generic reaction.

\section{Thermal decomposition of the primary fragments}

The preceding calculations showed that mid-chain and end-chain moieties of $\beta$-1,4-xylan can decompose in several fragments involving unimolecular concerted reactions. In addition, rate calculations have shown that the RE end-chain is much more reactive than the other parts of the polymer. The decomposition of RE end-chain leads to the formation of fragment 3 , which in turn decomposes to fragment 5 . In the following part of this study, we focused on the decomposition of important fragments. Accordingly, the thermal decomposition of fragment 5 is crucial to model the pyrolysis of $\beta-1,4-x y l a n$. Several unimolecular concerted reactions must be considered such as dehydration, glycosidic bond fissions, retro-adol, retro-ene, retro-Diels-Alder, keto-enol tautomerization, cyclic fragmentation or formation of a $\mathrm{C}_{5}$-ring. Scheme 11 depicts the three reactions involving the lowest calculated energy barriers. Ten other reactions have been investigated and are displayed in Scheme S2 in supplementary information. In addition, we have also taken into account the easiest glycosidic bond fission leading to the formation of fragment 1 (TS16). We calculated the corresponding rate constant since the fragment 5 involves an acyclic part, which makes it different from TS1. 


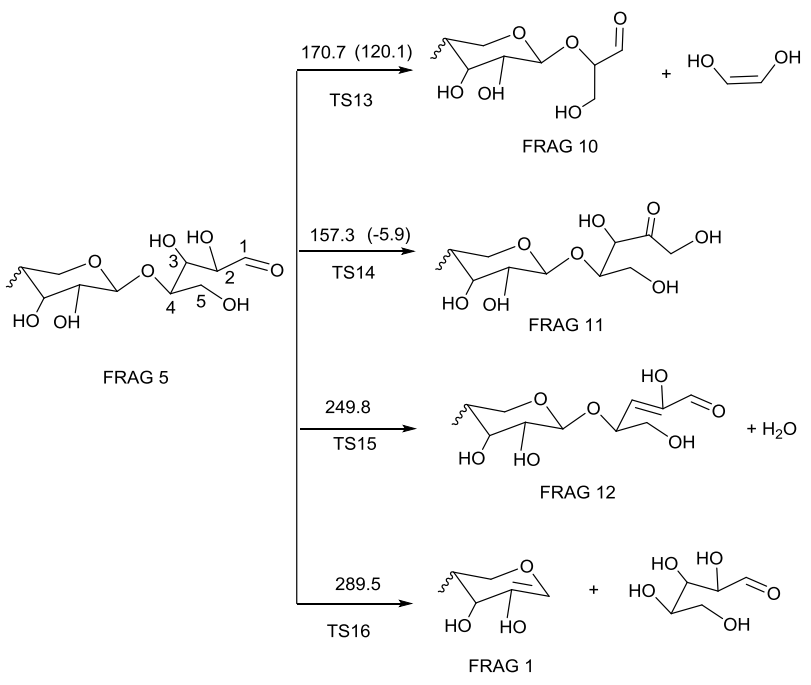

Scheme 11. Easiest reactions involved in the thermal decomposition of fragment 5. Barrier heights at $0 \mathrm{~K}$ (including ZPE) have been computed at the CBS-QB3 level of theory Values in parenthesis are reaction enthalpies at $0 \mathrm{~K}$ (in kJ mol$\left.{ }^{-1}\right)$.

Scheme 11 shows that two reactions have a low energy barrier. The first reaction involves a retro-aldol reaction via a 6-membered transition state (TS13) and leads to the formation of ethene-1,2-diol and a new fragment (FRAG10). This reaction is endothermic by around $120 \mathrm{~kJ} \mathrm{~mol}^{-1}$. Fragment 5 can also undergo a keto-enol isomerization (FRAG11) with a slightly lower energy barrier and a reaction enthalpy close to zero. Among all other reactions investigated for the acyclic moiety, dehydration between carbon atoms 2 and 3 has the lowest energy barrier $\left(249.8 \mathrm{~kJ} \mathrm{~mol}^{-1}\right)$. However, this value remains much higher than those involved in the two previous reactions. The last reactions considered in Scheme 11, is the glycosidic bond fission with an energy barrier slightly higher than the value computed for $\beta$-1,4xylan (Scheme 2). It is worth noting that the presence of the glycosidic bond in the model molecule is mandatory because it can prevent reactions in the acyclic part. For instance, Hu et al. ${ }^{12}$ studied the decomposition of xylose and put forward a 5-membered cyclization reaction involving a $\mathrm{H}$-atom shift from the hydroxyl group located on carbon atom \#4. At the B3LYP-D3 level of theory, this reaction has a low activation energy of $145.2 \mathrm{~kJ} \mathrm{~mol}^{-1}$. However, this reaction is impossible in $\beta-1,4$-xylan because the $\mathrm{O}-\mathrm{H}$ is in fact a glycosidic bond, as shown in fragment 5 (Scheme 11). Fig. 3 depicts the corresponding rate constants as a function of temperature. The rate coefficients are given in Table S1.5.

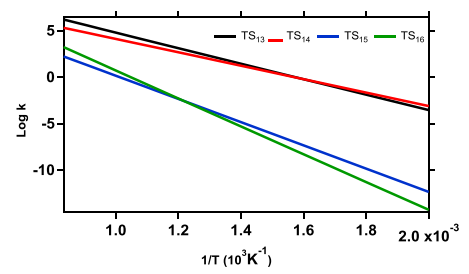

Figure 3: Computed rate constants $\left(\mathrm{s}^{-1}\right)$ involved in Scheme 11, versus the inverse temperature for T ranging from 500 to $1200 \mathrm{~K}$.

As expected, the two first reactions (TS13 and TS14) are largely predominant and fragments 10 and 11 can be considered as the main products formed from fragment 5, as well as ethene-1,2-diol. Even if entropic effects can favour the formation of TS15, reactions involving energy barrier above $250 \mathrm{~kJ} \mathrm{~mol}^{-1}$ cannot compete with retro-aldol or keto-enol isomerizations, especially for the temperature range considered in flash pyrolysis. This result is also consistent with the experimental observation of Yu et al. ${ }^{13}$ who detected a large amount of $\beta$-D-xylopyranosylglyceraldehyde (FRAG10) at the initial stage of the fast pyrolysis of xylobiose.

Fragments 10 and 11 are key structures in the pyrolysis of $\beta-1,4-x y l a n$ and we investigated possible pathways for their thermal decomposition. Scheme 12 depicts possible reactions of fragment 10. 


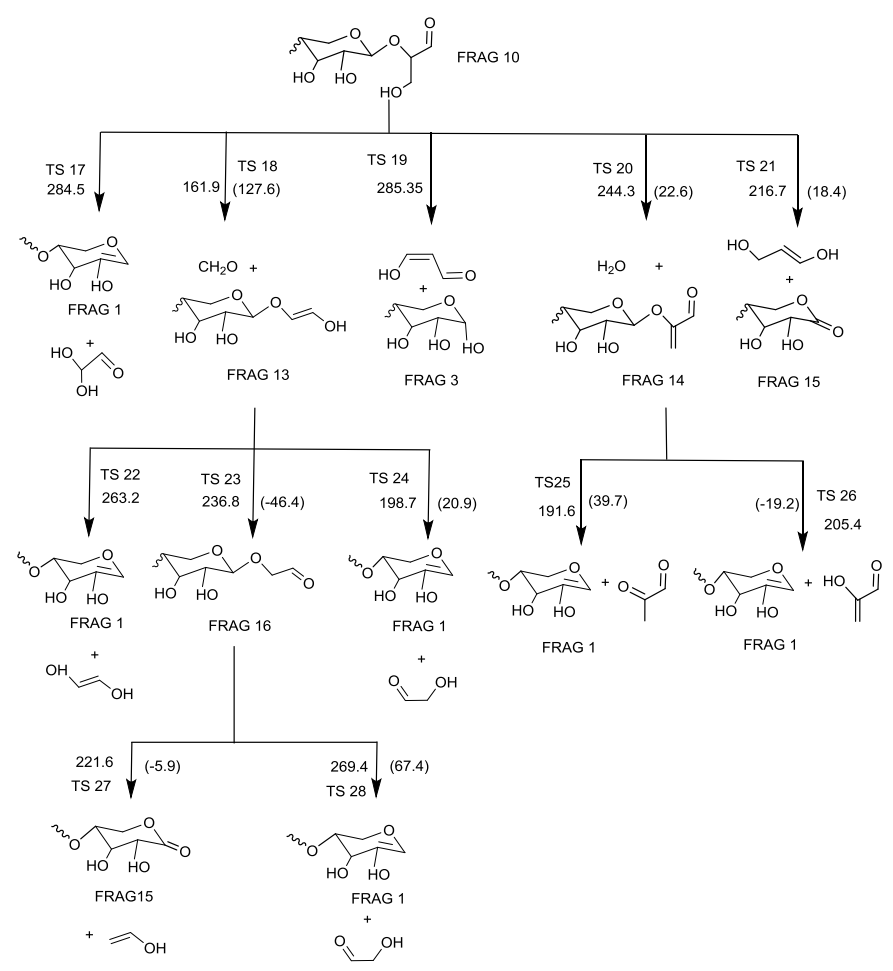

Scheme 12. Thermal decomposition pathways of fragment 10. Barrier heights at OK (including ZPE) have been computed at the CBS-QB3 level of theory. Values in parenthesis are reaction enthalpies at $0 \mathrm{~K}\left(\right.$ in $\left.\mathrm{kJ} \mathrm{mol}^{-1}\right)$.

The easiest route involves a retro-aldol reaction (TS18) yielding formaldehyde and a new fragment (FRAG13), which in turn undergoes a retro-ene reaction (TS24) to form hydroxyacetaldehyde (HAA, a main products observed experimentally ${ }^{12}$ ) and fragment 1 with a barrier of $198.7 \mathrm{~kJ} \mathrm{~mol}^{-1}$. The first reaction is endothermic by around $128 \mathrm{~kJ} \mathrm{~mol}^{-1}$. Another possible reaction of FRAG13 is the keto-enol tautomerization (TS 23) leading to fragment 16, despite an energy barrier higher by $38.1 \mathrm{~kJ} \mathrm{~mol}^{-1}$. All other pathways involved in the decomposition of fragment 10 have higher energy barriers. Thus, the 6-membered reaction yielding to fragment 15 and prop-1-ene-1,3diol (TS21) or the dehydration reaction leading to fragment 14 (TS20), lie 60.7 and $82.4 \mathrm{~kJ} \mathrm{~mol}^{-1}$, respectively, above the lowest energy barrier (TS18).

Fig. 4 depicts the main rate constants involved in the reactions of FRAG10, while Table S1.6 gives the rate coefficients for all the reactions mentioned above.

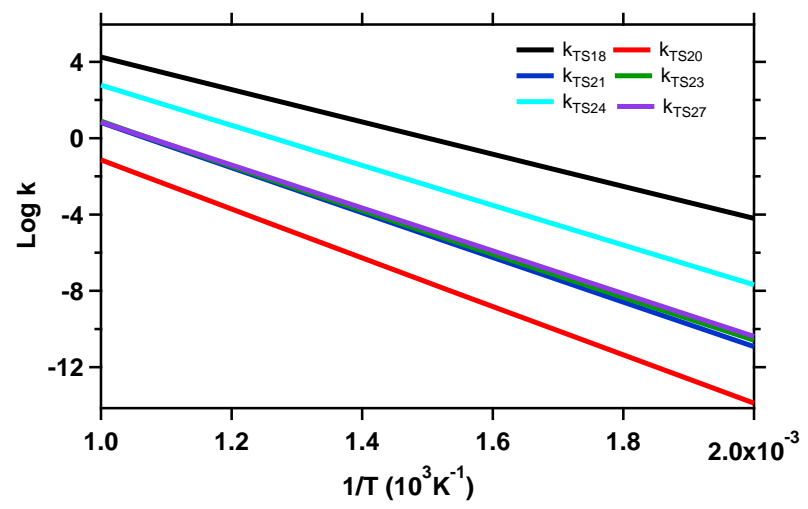

Fig. 4: Primary computed rate constants $\left(\mathrm{s}^{-1}\right)$ involved in Scheme 12, versus the inverse temperature for T ranging from 500 to $1000 \mathrm{~K}$.

Values of the rate constants are consistent with the trend observed for the energy barriers. The concerted elimination of formaldehyde leading to the formation of fragment 13 is largely dominant while dehydration remains the most difficult process, as previously observed. Fig. 4 also confirms that fragment 1 and hydroxyacetaldehyde will be the main products of the thermal decomposition of fragment 10, since the formation of fragment 16 is negligible compared to the former products (factor 200 at $750 \mathrm{~K}$ ).

The pyrolysis mechanism of fragment 11, the other principal decomposition product of fragment 5 (Scheme 11), has also been thoroughly investigated and two low energy pathways have been highlighted (Scheme 13). 


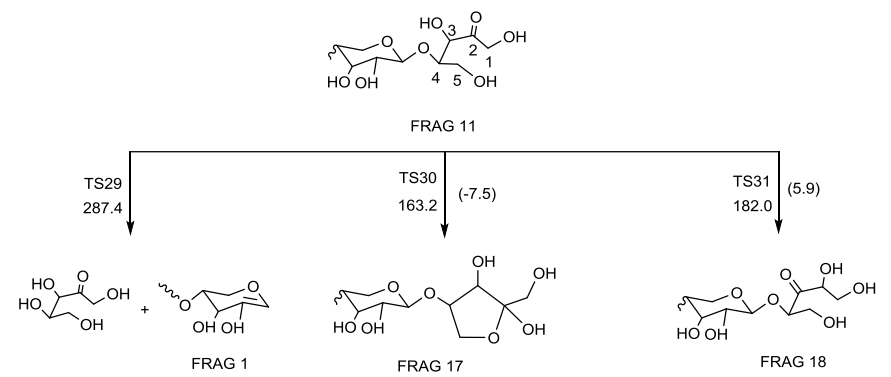

Scheme 13. Thermal decomposition pathways of fragment 11. Barrier heights at $0 \mathrm{~K}$ (including ZPE) have been computed at the CBS-QB3 level of theory. Values in parenthesis are reaction enthalpies at $0 \mathrm{~K}\left(\right.$ in $\left.\mathrm{kJ} \mathrm{mol}^{-1}\right)$.

The easiest reaction (TS30) is a ring closure to form a tetrahydrofuran type structure with a low barrier of $163.2 \mathrm{~kJ}^{\mathrm{mol}}{ }^{-1}$. The corresponding transition state involves both the formation of a bond between carbon \#2 and the oxygen atom bonded to carbon \#5 and a shift of the $\mathrm{H}$-atom of the hydroxyl group towards the O-atom of the ketone function (Scheme 14).

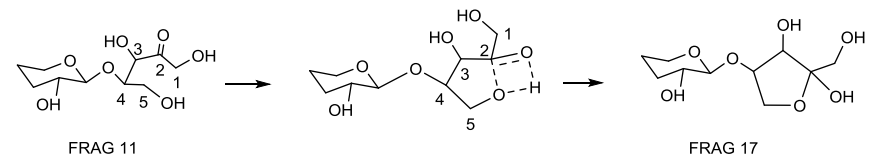

Scheme 14. Reactions involved in the ring closure of fragment11, with the model molecule used to perform the CBS-QB3 calculation

The second lowest energy pathway (TS 31) is an isomerization leading to fragment 18 (Scheme 13). The energy barrier is slightly higher than the previous one $\left(182.0 \mathrm{~kJ} \mathrm{~mol}^{-1}\right)$. It is worth noting that these two reactions are almost athermal but entropic effects disfavour the formation of the fragment 17 due to the ring closure. The rate constants showed in Fig. 5 confirms this statement. The formation of the $C_{5}$ ring is slightly easier at low temperature than the isomerization (due to a lower energy barrier), while a reverse trend is observed at high temperature (loss of entropy in TS30). Table S1.7 gives the corresponding rate coefficients.

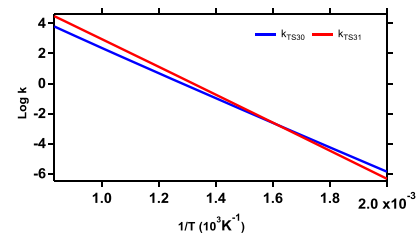

Fig. 5: Computed rate constants $\left(\mathrm{s}^{-1}\right)$ involved in Scheme 13 (fragment 11), versus the inverse temperature for T ranging from 500 to $1200 \mathrm{~K}$.

For consistency, we also considered the most favourable glycosidic bond fission (TS29) leading to fragment 1. However, the high activation barrier prevents any kinetic effect of this reaction in the thermal degradation of fragment 11.

Considering all the lowest energy pathways, up to the formation of fragment $10, \beta-1,4$-xylan decomposes to several polymer structures, which in turn react mainly to form a particular fragment, namely fragment 1 . The reactions of fragment 11 brings up two new structures (FRAG 17 and 18) for which detailed decomposition reactions have been investigated.

The reactions of fragment 17 (Scheme S3 in the supplementary information), involve glycosidic bond fissions or dehydrations. However, the energy barriers computed are all above $280 \mathrm{~kJ} \mathrm{~mol}^{-1}$ and cannot compete with the reverse reaction yielding fragment 11 (ring opening) or with reactions of fragment 18 (see further). In particular, the formation of furfural is often presented in the literature ${ }^{7,8,12,29}$ by a sequence of reactions involving successive dehydrations. Moreover, Hu et al. ${ }^{12}$ experimentally detected furfural, during the fast pyrolysis of xylose and xylobiose, while only smaller amount has been observed with xylan. In our study, furfural formation can be represented by the following reactions (Scheme 15):

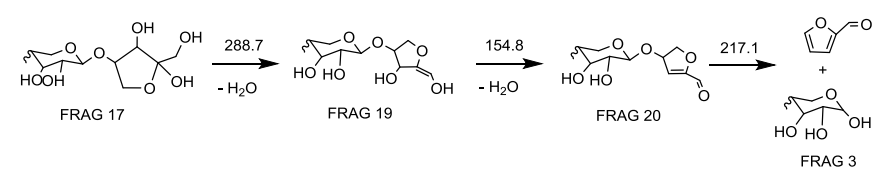

Scheme 15. Furfural formation from fragment 17. Barrier heights at $0 \mathrm{~K}$ (including ZPE) have been computed at the CBS-QB3 level of theory (in $\mathrm{kJ}^{\mathrm{mol}}{ }^{-1}$ ). 
We investigated all possible dehydrations of FRAG17 and the lowest energy barrier found reaches $288.7 \mathrm{~kJ} \mathrm{~mol}^{-1}$ (Scheme 15 ), in correct agreement with previous studies performed at the M062X level of theory ${ }^{6,8,29}$. Other pathways investigated in the literature ${ }^{8,11,12}$ consider first dehydrations of the fragment 3 (or an equivalent structure in the case of xylose as molecule model), followed by a ring closure to form furfural. Once again, the dehydration steps involve high energy barriers. Therefore, it seems difficult to explain the formation of furfural by such a mechanism of successive molecular eliminations and this result raises the issue of its formation from unimolecular concerted reactions.

On the other hand, the thermal degradation of fragment 18 can occur with lower energy pathways, as shown in Scheme 16.

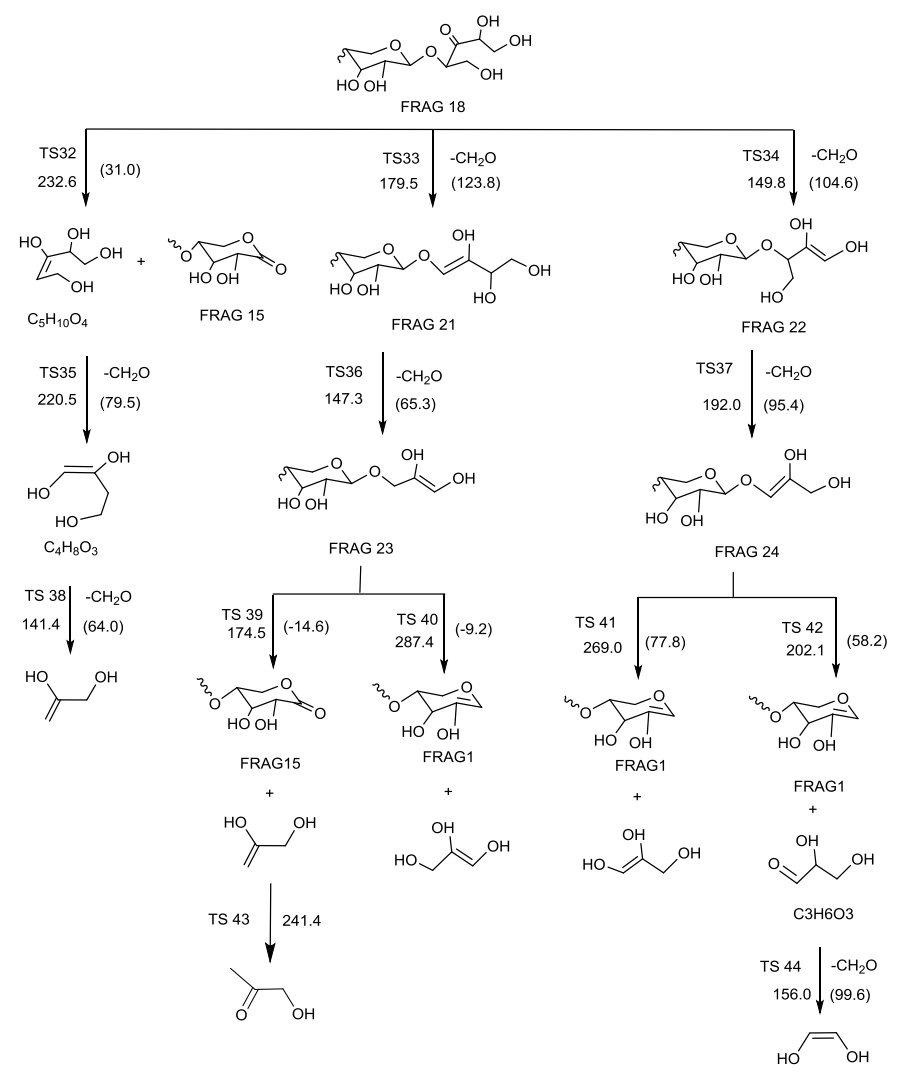

Scheme 16. Thermal decomposition pathways of fragment 18. Barrier heights at $0 \mathrm{~K}$ (including ZPE) have been computed at the CBS-QB3 level of theory. Values in parenthesis are reaction enthalpies at $0 \mathrm{~K}$ (in $\mathrm{kJ} \mathrm{mol}-1$ ).

Indeed, this fragment can react easily by retro-aldol reactions leading to the formation of formaldehyde and two possible fragments (FRAG 21 and 22) with barrier heights equal to 179.5 and $149.8 \mathrm{~kJ} \mathrm{~mol}^{-1}$, respectively. These fragments undergo similar retro-aldol reactions to form smaller fragments (FRAG 23 and 24) which in turn react by glycosidic bond fissions involving 4 or 6-membered concerted reactions. All these reactions lead to fragments 1 or 15, already encountered, as well as light species such as prop-2-ene-1,2-diol, glyceraldehyde $\left(\mathrm{C}_{3} \mathrm{H}_{6} \mathrm{O}_{3}\right)$ and its tautomer, the dehydroglycerol (pro-1-ene-1,2,3-triol). Glyceraldehyde has been reported in many flash pyrolysis of xylan or hemicellulose ${ }^{3,9,12}$. However, this species can easily react by a retro-aldol reaction (TS44) to form ethenediol and formaldehyde.

Another possible decomposition pathway of fragment 18 , is a 6-membered concerted reaction yielding to pent-3-ene-1,2,3,5-tetrol $\left(\mathrm{C}_{5} \mathrm{H}_{10} \mathrm{O}_{4}\right)$ and fragment 15. This reaction is, at least, $53 \mathrm{~kJ} \mathrm{~mol}^{-1}$ above the retro-aldol reactions and it should represent a minor route for the decomposition of FRAG18. All the other pathways investigated in this work, led to larger energy barriers. Fig. 6a compares the rate constants involve in the primary steps of the thermal decomposition of fragment 18. 


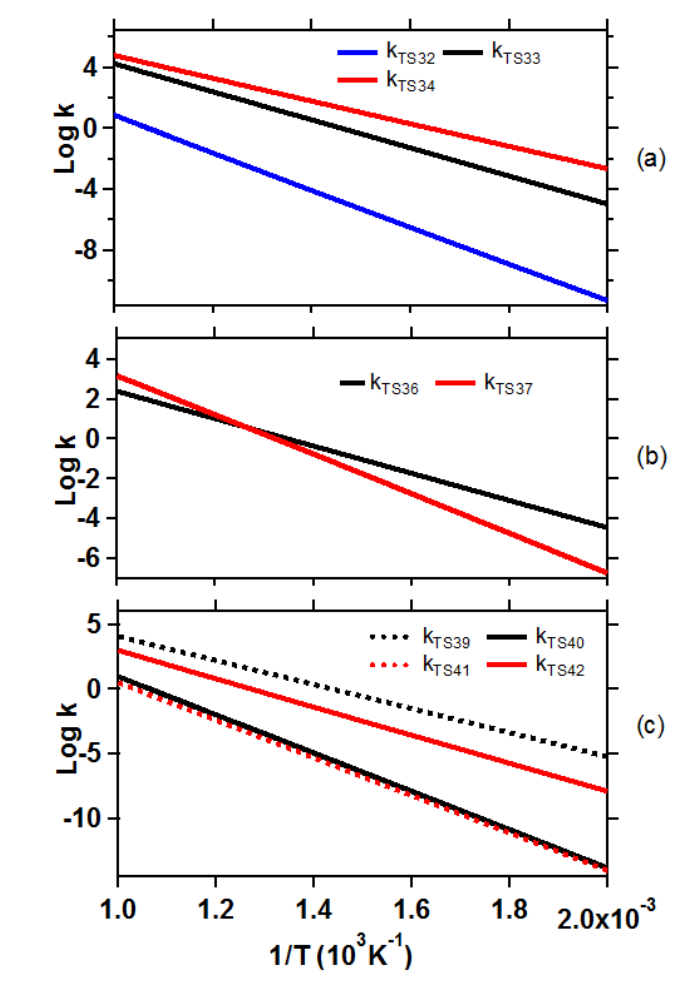

Fig. 6: Computed rate constants $\left(\mathrm{s}^{-1}\right)$ involved in Scheme 19, versus the inverse temperature for T ranging from 500 to $1200 \mathrm{~K}$.

As expected, the glycosidic bond fission (TS32) cannot compete with the retro-aldol reactions. Concerning these latter, the rate of formation of FRAG22 (TS34) is ten times faster than that of FRAG21 (TS33) at 750K, due to a lower energy barrier. However, the stronger hydrogen bonds (due to hydroxyl groups) in TS34 reduce the entropic contribution of internal rotations, which decrease the difference observed with temperature (Figure 6a). The same phenomenon can be observed for the reactions of fragments 21 and 22 (Figure 6b). Despite a difference of $44.8 \mathrm{~kJ} \mathrm{~mol}^{-1}$ between the two pathways, the formation of fragment 24 becomes more favourable at high temperature due to strong hydrogen bonds in TS36, as illustrated in Scheme 17. All the rate coefficients involved in Fig. 6 are given in Table S1.8.

While the importance of fragment 1 in the pyrolysis of xylan has already been discussed, fragment 15 is formed during the thermal decomposition of FRAG10 and FRAG18, and it appears to be a new key compound. In the following, we investigated the main decomposition routes of these two key fragments.

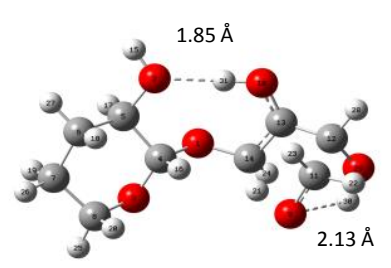

TS 36

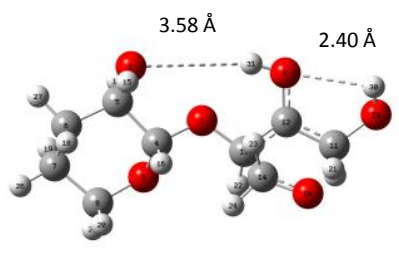

TS 37

Scheme 17: Optimized structures (B3LYP/6-311++G(d,p)) of TS36 and TS37 involved in the decomposition of FRAG18 with hydrogen bonds represented by dotted lines.

\section{Thermal decomposition of Fragment 15}

Scheme 18 shows the lowest energy pathways found to model the pyrolysis of fragment 15 through concerted reactions. 


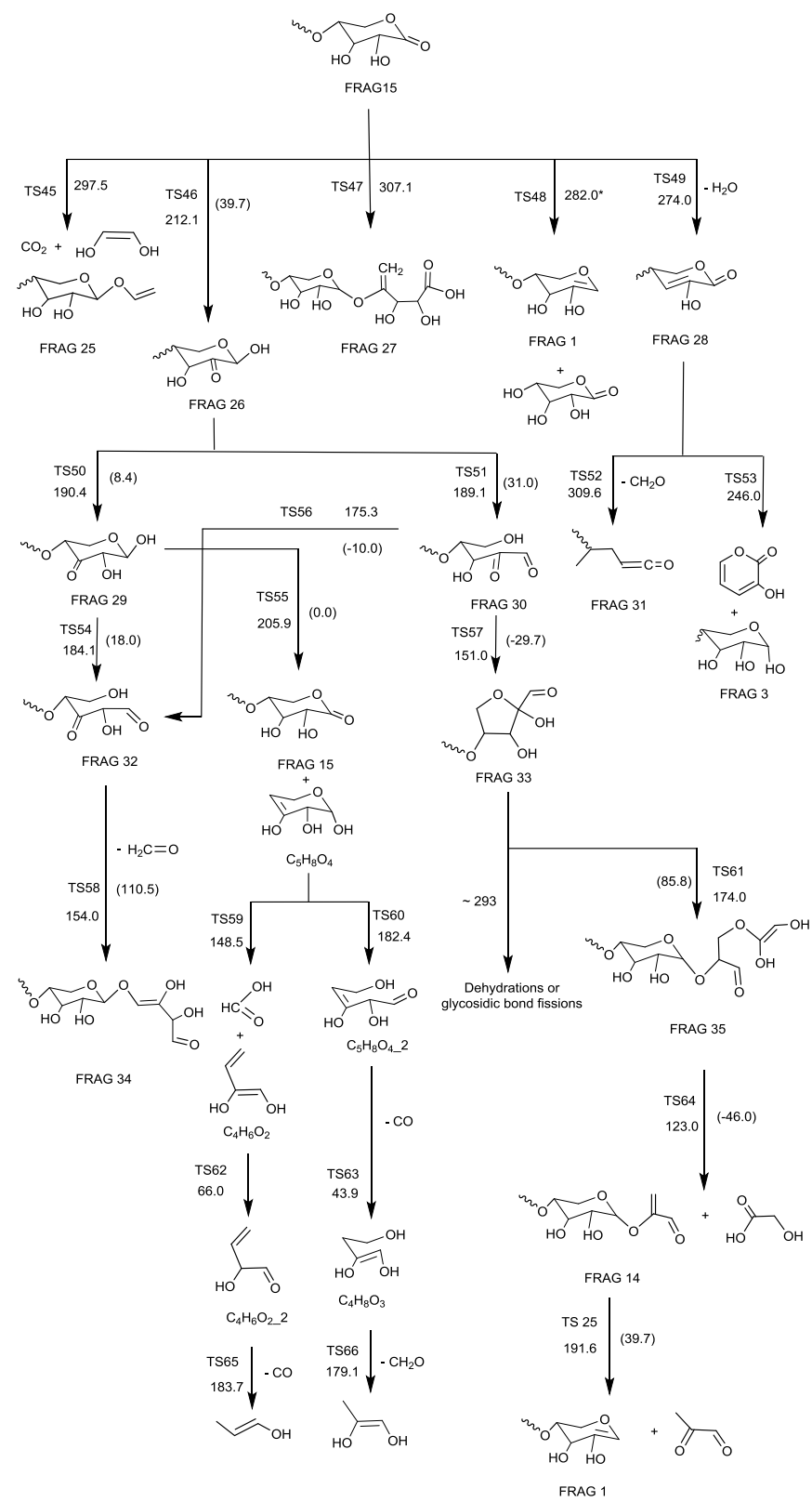

Scheme 18. Thermal decomposition pathways of fragment 15. Barrier heights at $0 \mathrm{~K}$ (including ZPE) have been computed at the CBS-QB3 level of theory. Values in parenthesis are reaction enthalpies at $0 \mathrm{~K}$ (in $\mathrm{kJ} \mathrm{mol}-1)$.

The lowest energy barrier implies a shift of the carbonyl group by internal H-atom transfers to form fragment 26 (TS46). The direct elimination of $\mathrm{CO}_{2}$ (TS45) or the ring opening to form fragment 27 (TS47), require very high activation energies and should not be competitive. In the same way, reactions such as glycosidic bond fission (TS48) or dehydration (TS49) cannot compete with the isomerization (TS46). From fragment 26, two decomposition routes can be highlighted. The first one involves another shift of the carbonyl group and leads to the formation of fragment 29 , which in turn can undergo a ring opening yielding fragment 32 . This latter compound can react by an easy retro-aldol reaction forming formaldehyde and a new fragment (FRAG34). All the energy barriers are below $191 \mathrm{~kJ} \mathrm{~mol}^{-1}$, which provides a promising pathway for the decomposition of fragment 15 . An alternative decomposition of fragment 29 is the formation of the lactone structure corresponding to fragment 15 and a dihydropyran compound, which can decompose to form light species such as $\mathrm{CO}$, formic acid or formaldehyde (Scheme 18).

The second decomposition of fragment 26 is a direct ring opening to form fragment 30 , which can easily undergo a $C_{5}$ ring closure to form a tetrahydrofuran compound (FRAG33), or react by a keto-enol isomerization (TS56) to yield fragment 32. The energy barrier of the latter is around $25.1 \mathrm{~kJ} \mathrm{~mol}^{-1}$ higher than that involved in the $\mathrm{C}_{5}$ ring closure but the lower number of internal rotation loss in the transition state could promote the activation entropy. Moreover, even if the formation of the $C_{5}$ ring is thermodynamically favourable $\left(\Delta_{\mathrm{r}} \mathrm{H}_{0 \mathrm{~K}}=-29.7 \mathrm{~kJ}\right.$ mol ${ }^{1}$ ), the subsequent dehydration reactions to form furfural require high energy barriers, as previously discussed. An easier reaction is the ring opening to form fragment 35 (TS61), with a barrier height of $174 \mathrm{~kJ} \mathrm{~mol}^{-1}$. This fragment can easily decompose by a retro-ene reaction, as shown in Scheme 19. 


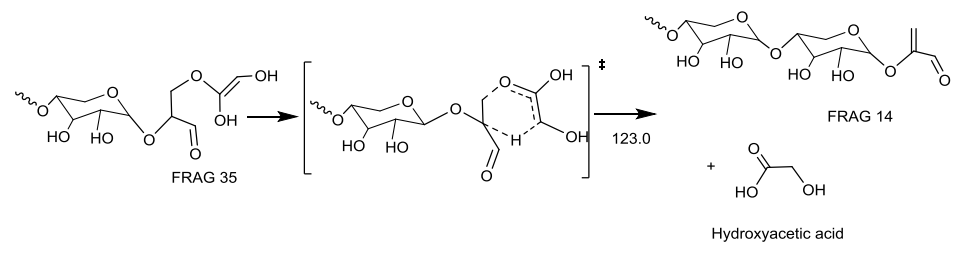

Scheme 19. Retro-ene reaction involved in the thermal decomposition of fragment 35 and related to Scheme 18.

This reaction leads to the formation of hydroxyacetic acid and the fragment 14, already encountered in the decomposition of FRAG10 (Scheme 12). The lower decomposition channel found for fragment 14 (TS 25) is a retro-ene reaction leading to the formation of methylglyoxal and fragment 1.

The decomposition of the fragment 15 leads to a complex decomposition scheme and it can be interesting to highlight the reactions having a kinetic effect on the consumption of this fragment. Fig. 7 depicts the rate constants of the main reactions, as a function of the temperature (the corresponding rate coefficients are given in Table S1.9). For complex reaction sequences like those involved in Scheme 18 , relations between forward rate constants and the kinetic importance of the corresponding reactions have to be analysed with caution, due to the kinetic influence of the reverse reactions. For this purpose, the potential energy profile involved in the decomposition of FRAG15 in presented in Scheme 20. Exit channels correspond to fragmentation reactions leading to the formation of two products.

Fig. 7a shows that the formation of FRAG29 (TS50) is slightly easier than the formation of FRAG30 (TS51). Starting from these two fragments, Scheme 20 shows that the formation of FRAG33 (TS57) implies a lower energy barrier than those involved in the formation of FRAG32 (TS54 and TS56) or the exit channel leading to FRAG15 and $\mathrm{C}_{5} \mathrm{H}_{8} \mathrm{O}_{4}$ (TS55). However, the corresponding rate constants, depicted in Fig. 7b show that the formation of FRAG32 from TS54 competes with the formation of FRAG33 (TS57) and is promoted at high temperature. This result can be explained by a negative activation entropy involved in the formation of FRAG33 and due to the ring closure.

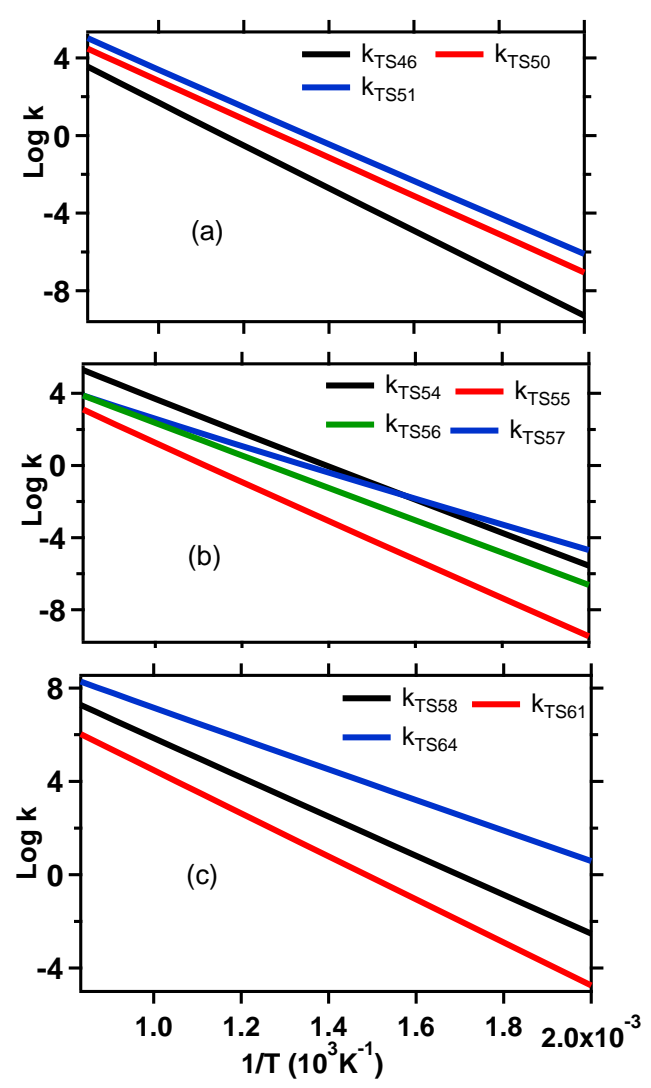

Fig. 7. Computed rate constants $\left(\mathrm{s}^{-1}\right)$ involved in Scheme 18 (fragment 15), versus the inverse temperature for T ranging from 500 to $1200 \mathrm{~K}$. Only the main channels have been depicted on this figure.

This entropic effect disfavours the reaction at high temperature compared to the ring opening involved in the formation of FRAG32. Fig. 7c depicts the rate constants for the reactions of fragments 32 and 33 and leading to the exit products. 


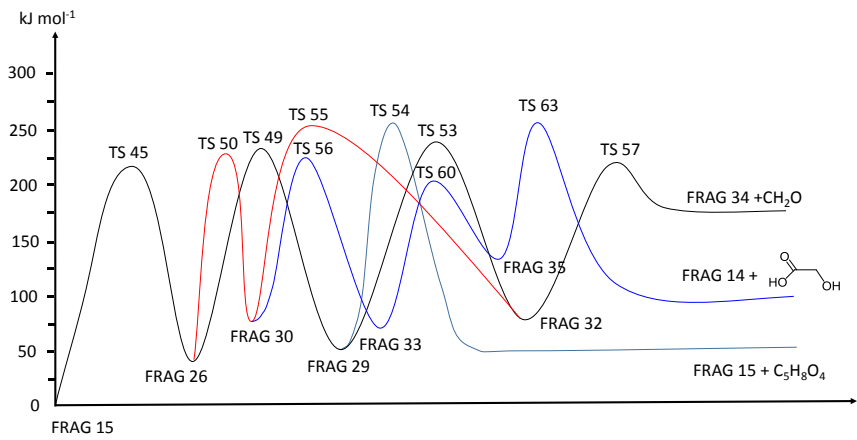

Scheme 20. Potential energy profile involved in the main unimolecular reactions of FRAG15. Energies are given at $0 \mathrm{~K}$ in $\mathrm{kJ} \mathrm{mol}^{-1}$.

The thermal decomposition of FRAG33 involves a two-step mechanism with the formation of FRAG35 as intermediate. Fig. 7c shows that the formation of FRAG34 (TS58) and formaldehyde is largely promoted over the formation of FRAG35 (TS61) by, at least, a factor of ten. Moreover, if the decomposition of the latter involves a low energy barrier (TS64), the reverse reaction remains largely favoured compared to the exit channel leading to FRAG14 and hydroxyl-acetic acid (Scheme 20). These results show that the thermal decomposition of fragment 15 should mainly lead to the formation of fragment 34 . In the following, we focus on its decomposition pathways. Several reactions of low energy barriers have been identified and are summarized in Scheme 21.

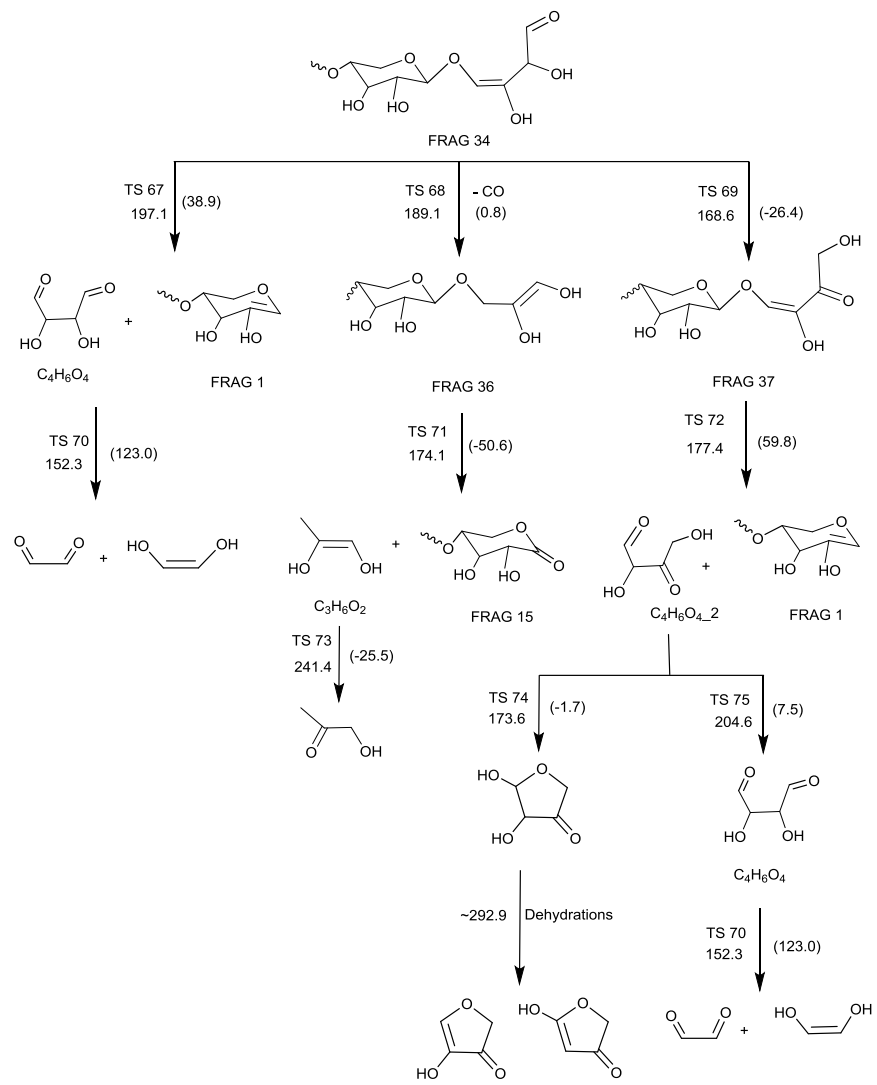

Scheme 21. Thermal decomposition pathways of fragment 34. Barrier heights at $0 \mathrm{~K}$ (including ZPE) have been computed at the CBS-QB3 level of theory. Values in parenthesis are reaction enthalpies at $0 \mathrm{~K}$ (in $\mathrm{kJ} \mathrm{mol}-1)$.

Three primary reactions have been identified with barrier heights below $209.2 \mathrm{~kJ} \mathrm{~mol}^{-1}$. The first reaction (TS 67) is a retro-ene reaction involving a glycosidic bond fission. This reaction leads to the formation of fragment 1 and the 2,3-dihydroxybutanedial $\left(\mathrm{C}_{4} \mathrm{H}_{6} \mathrm{O}_{4}\right)$ which in turn can easily decompose by retro-aldol fragmentation yielding glyoxal and ethenediol. The second reaction (TS68) is a CO elimination via a concerted reaction shown in Scheme 22:

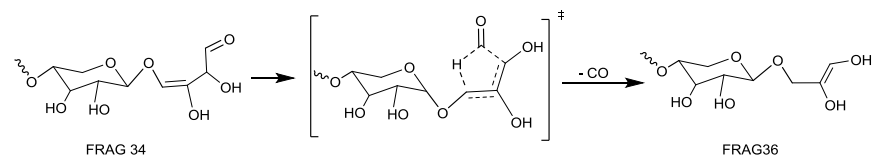


Scheme 22. CO elimination in fragment 34 .

Carbon monoxide is an important product observed during the pyrolysis of hemicellulose ${ }^{3}$. The barrier height computed is only $189.1 \mathrm{~kJ}$ $\mathrm{mol}^{-1}$, making it an attractive route for producing CO. The last reaction considered is the internal isomerization (TS 69) between the carbonyl group and the nearest hydroxyl group. It involves the simultaneous shift of two hydrogen atoms.

Fragment 36 mainly reacts by a retro-ene reaction involving a glycosidic bond fission (TS71) to form the lactone structure (FRAG15) and an isomer of the methylglyoxal $\left(\mathrm{C}_{3} \mathrm{H}_{6} \mathrm{O}_{2}\right)$. Fragment 37 decomposes by a similar reaction but the two products formed are the fragment 1 (with one less monomer) and the 2,4-dihydroxy-3-oxobutanal $\left(\mathrm{C}_{4} \mathrm{H}_{6} \mathrm{O}_{4} 2\right)$. This compound can react by $\mathrm{C}_{5}$ ring closure (TS74) to produce 4,5dihydroxydihydrofuran-3-one or by isomerization leading to the formation of 2,3-dihydroxybutanedial, previously encountered in the decomposition of FRAG34 (TS67). Again, the formation of the $C_{5}$ ring requires a low activation energy compared to isomerization. However, this cycle cannot easily react by dehydrations (or other unimolecular concerted reactions) so, it can only accumulate or react by the reverse reaction. On the other hand, the 2,3-dihydroxybutanedial can undergo a retro-aldol reaction with a low energy barrier (152.3 $\mathrm{kJ} \mathrm{mol}^{-1}$ ) to form glyoxal and ethenediol.

Fig. 8 depicts the rate constants as a function of temperature (the corresponding rate coefficients are given in Table S1.10).

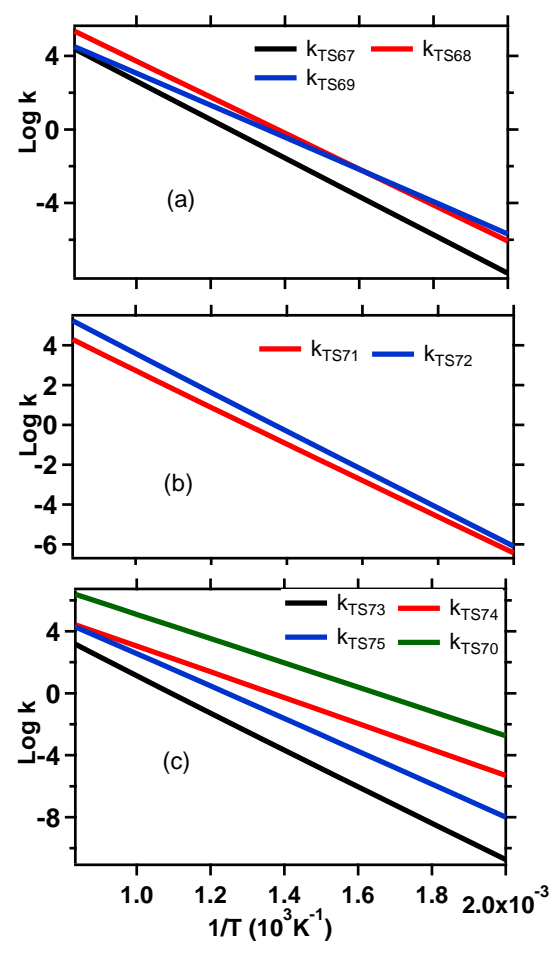

Fig. 8. Computed rate constants $\left(\mathrm{s}^{-1}\right)$ involved in Scheme 21 (fragment 34), versus the inverse temperature for T ranging from 500 to $1200 \mathrm{~K}$.

Fig. $8 \mathrm{a}$ and $8 \mathrm{~b}$ show that the two reaction routes leading to the formation of fragments 1 and 15 (via TS 68 and 69) have a kinetic influence in the thermal decomposition of fragment 34. For temperatures higher than $600 \mathrm{~K}$, the formation of fragment 36 (and a fortiori fragment 15) will be slightly favoured. Indeed, the elimination of carbon monoxide (TS68) leads to a positive activation entropy due to the fragmentation process unlike the isomerization reaction (TS69) for which the hydrogen shift involved a loss of entropy in the TS. Even if the energy barrier for the latter reaction is lower, it cannot compensate this entropic effect.

In summary, the unimolecular decomposition of $\beta-1,4-x y l a n$ can occur by glycosidic bond fission of the mid-chains, leading, mainly, to the formation of fragments 1 and 3. The latter compound reacts in the same way as the reductive chain ends, i.e., by ring opening to form a new structure, which in turn leads to subsequent decompositions with the formation of several fragments. Our calculations have shown that all the low energy pathways lead to the formation of fragment 1 and, to a lesser extent, to fragment 15 (with light species). Fragment 15 decomposes in several ways, leading again to the formation of structures similar to fragment 1 and fragment 15, involving an iterative process. The last structure to be taken into account to correctly describe the thermal decomposition of 1,4- $\beta$-xylan from unimolecular reactions is the fragment 1 . This fragment is a key structure, since it systematically appears in the most likely decomposition pathways of the other fragments.

\section{Thermal decomposition of Fragment 1}




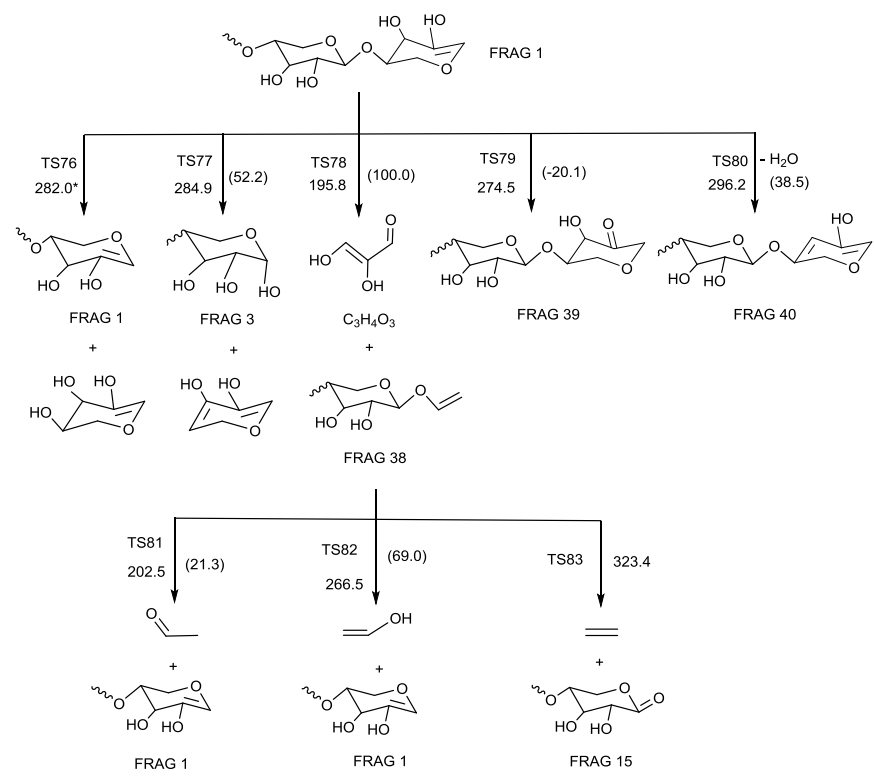

Scheme 23. Thermal decomposition pathways of fragment 1 . Barrier heights at $0 \mathrm{~K}$ (including ZPE) have been computed at the CBS-QB3 level of theory. Values in parenthesis are reaction enthalpies at $0 \mathrm{~K}$ (in $\mathrm{kJ} \mathrm{mol}-1)$.

Glycosidic bond fissions (TS76 and 77) and dehydrations (TS80), have been considered, but the energy barriers are similar to those previously computed for the other fragments and prevent any kinetic influence during the pyrolysis of fragment 1 . In the same way, the keto-enol isomerization (TS 79) requires a high activation energy due, especially, to cyclic constraints. The lowest energy pathway found is a retro Diels-Alder reaction leading to fragment 38 and 2,3-dihydroxyprop-2-enal $\left(\mathrm{C}_{3} \mathrm{H}_{4} \mathrm{O}_{3}\right)$ as described in Scheme 24.

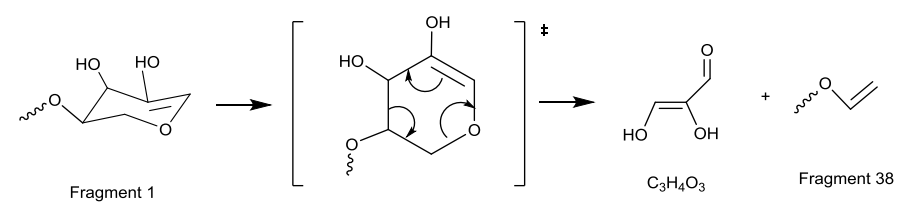

Scheme 24. Retro Diels-Alder reaction involved in the thermal decomposition of fragment1

This reaction is often omitted in theoretical studies of reactions of hemicellulose since many works use xylose as model molecule or only investigate the primary decomposition channels of xylobiose. In their theoretical study on the pyrolysis mechanism of xylobiose, Li et al. ${ }^{8}$ considered a similar retro Diels-Alder reaction. At the $M 062 X / 6-31+G(d, p)$ level of theory, they computed an energy barrier equal to 210.5 $\mathrm{kJ} \mathrm{mol}^{-1}$, in agreement with the value of $195.8 \mathrm{~kJ} \mathrm{~mol}^{-1}$ obtained in this work. The fragment 38 obtained, has a short linear chain, which reduce the number of possible reactions. The lowest energy barrier found is related to the retro-ene reaction (TS81) leading to acetaldehyde and a new structure similar to fragment 1 , but reduced by one monomer. Two other possible reactions involve a 4membered concerted reaction (TS82 and TS83). However, the energy barriers are significantly higher and cannot compete with the retroene reaction.

While acetaldehyde is a main product observed experimentally during the pyrolysis of $x \operatorname{lan}^{3}$, the formation of 2,3-dihydroxyprop-2-enal is not cited. Hu et al. ${ }^{12}$ reported a significant formation of a species having the same mass, during the pyrolysis of xylan, while it does not appear in the case of xylose or xylobiose. They affected the peak observed by gas chromatography, to 1-hydroxy-2-butanone. From our analysis, it seems difficult to explain the formation of a saturated $C_{4}$ linear chain by unimolecular concerted reactions deriving from $\beta$-1,4xylan. Moreover, to our knowledge, the formation of 1-hydroxy-2-butanone is never explained by the mechanisms proposed in the literature. We investigated possible reaction pathways for the decomposition of 2,3-dihydroxyprop-2-enal. The lower energy path found is depicted in Scheme 25 .

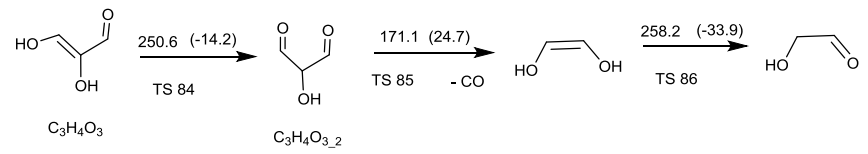

Scheme 25. Reaction pathway involved in the thermal decomposition of 2,3-dihydroxyprop-2-enal. Barrier heights at $0 \mathrm{~K}$ (including ZPE) have been computed at the CBS-QB3 level of theory. Values in parenthesis are reaction enthalpies at $0 \mathrm{~K}^{(\mathrm{in} \mathrm{kJ} \mathrm{mol}}{ }^{-1}$ ). 
2,3-dihydroxyprop-2-enal can react by keto-enol tautomerization to form hydroxypropendial, with an energy barrier close to $250 \mathrm{~kJ} \mathrm{~mol}^{-1}$ (TS84). The hydroxypropanedial formed can easily decompose by CO elimination (TS 85) to form ethenediol, which in turn isomerizes by another keto-enol tautomerization to form hydroxyacetaldehyde. The barrier height of this last reaction is equal to $258.2 \mathrm{~kJ} \mathrm{~mol}^{-1}$ in agreement with the value found for TS84. Fig.9 depicts the rate constants related to Scheme 23 (rate coefficients are given in Table S1.11). According to Fig. 9 the retro Diels-Alder reaction is the most likely decomposition route for the fragment 1 . Other processes cannot compete in the temperature range considered. Likewise, fragment 38 entirely reacts by retro-ene reaction to form acetaldehyde and a new fragment 1 even though the rate constant is 44 times lower than the retro Diels-Alder reaction at $750 \mathrm{~K}$. It may be explained by the difference between activation energies, but more importantly by the number of internal rotations frozen in the transition states. Indeed, the retro Diels-Alder reaction involves no loss of internal rotation and the entropy of the transition state is higher than the reactant, due to the ring opening process. Conversely, the TS involved in the retro-ene reaction has two frozen rotations, which lower its entropy. Finally, Fig. $9 \mathrm{~b}$ shows that unimolecular keto-enol tautomerizations require high activation energy and are rate-controlling steps.

Finally, a new iterative process involving the fragment 1 emerges from Scheme 23. This result is particularly interesting since fragment 1 is a key fragment in the thermal degradation of $\beta-1,4-x y l a n$. The regeneration of a similar structure permits to decrease the length of the polymer chain without changing the nature of the reactions involved. Indeed, fragment 1 is consumed by a retro Diels-Alder reaction (TS78), but a similar structure having one less monomer is produced by a retro-ene reaction (TS81). This reaction sequence can be compared to chain-propagating step, even if no radical or ion is involved in the mechanism.

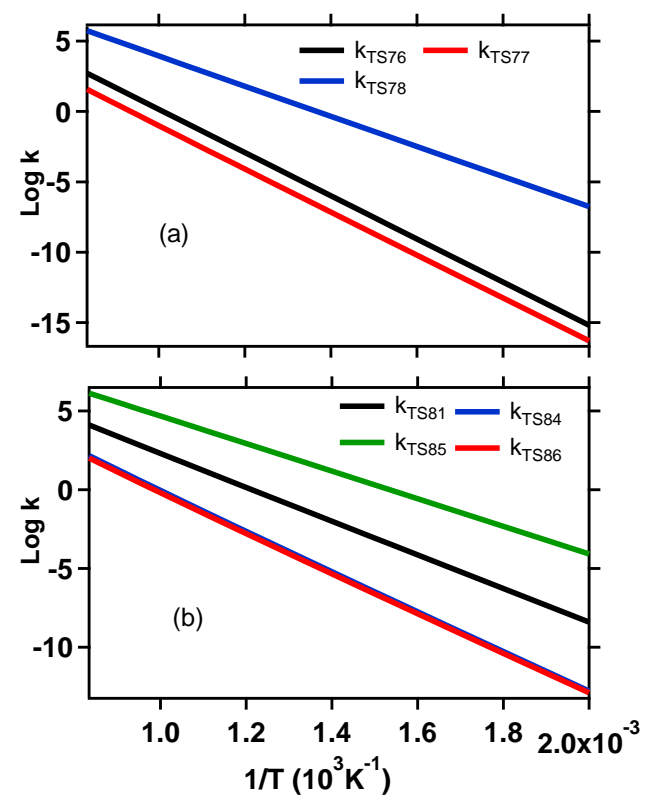

Fig. 9. Computed rate constants $\left(\mathrm{s}^{-1}\right)$ involved in the thermal decomposition of fragment 1 versus the inverse temperature for T ranging from 500 to $1200 \mathrm{~K}$.

Schemes 26 and 27 summarize the mechanism proposed for the thermal decomposition of $\beta-1,4-x y l a n$ by unimolecular concerted reactions. Based on previous rate calculations and reaction enthalpies, we selected the lower energy pathways. All the energies retained in this simplified mechanism are below $213.4 \mathrm{~kJ} \mathrm{~mol}^{-1}$, excepted for the initial glycosidic bond fission (occurring only in mid-chain decomposition).

Reductive end-chain is the easiest primary reaction of $\beta-1,4$-xylan and leads after successive elementary reactions to the formation of fragments 1 and 15. Fragment 1 is the most important one and undergoes a two-step decomposition in a closed sequence mechanism, restoring a similar structure as FRAG1 but containing one less monomer. As mentioned previously, fragment 15 has a weaker kinetic influence on the thermal decomposition of $\beta-1,4$-xylan but it cannot be neglected a priori. Its thermal decomposition involves two main pathways. The first one leads to the formation of fragment 1 , while the second pathway again provides a similar structure as fragment 15 with one less monomer, involving a new closed sequence mechanism.

Among all the products experimentally observed during the pyrolysis of hemicellulose, many studies ${ }^{5,11,12,30-32}$ have detected and quantified important species contained in gas phase and bio-oils. However, the structures of hemicelluloses are more complex than that of $\beta-1,4-x y l a n$ and the presence of minerals can strongly modify the nature and the quantity of the products formed. In addition, commercial xylan always contains residues (usually less than $10 \%$ ) which makes difficult a direct comparison of the species observed with those predict by a model molecule. 


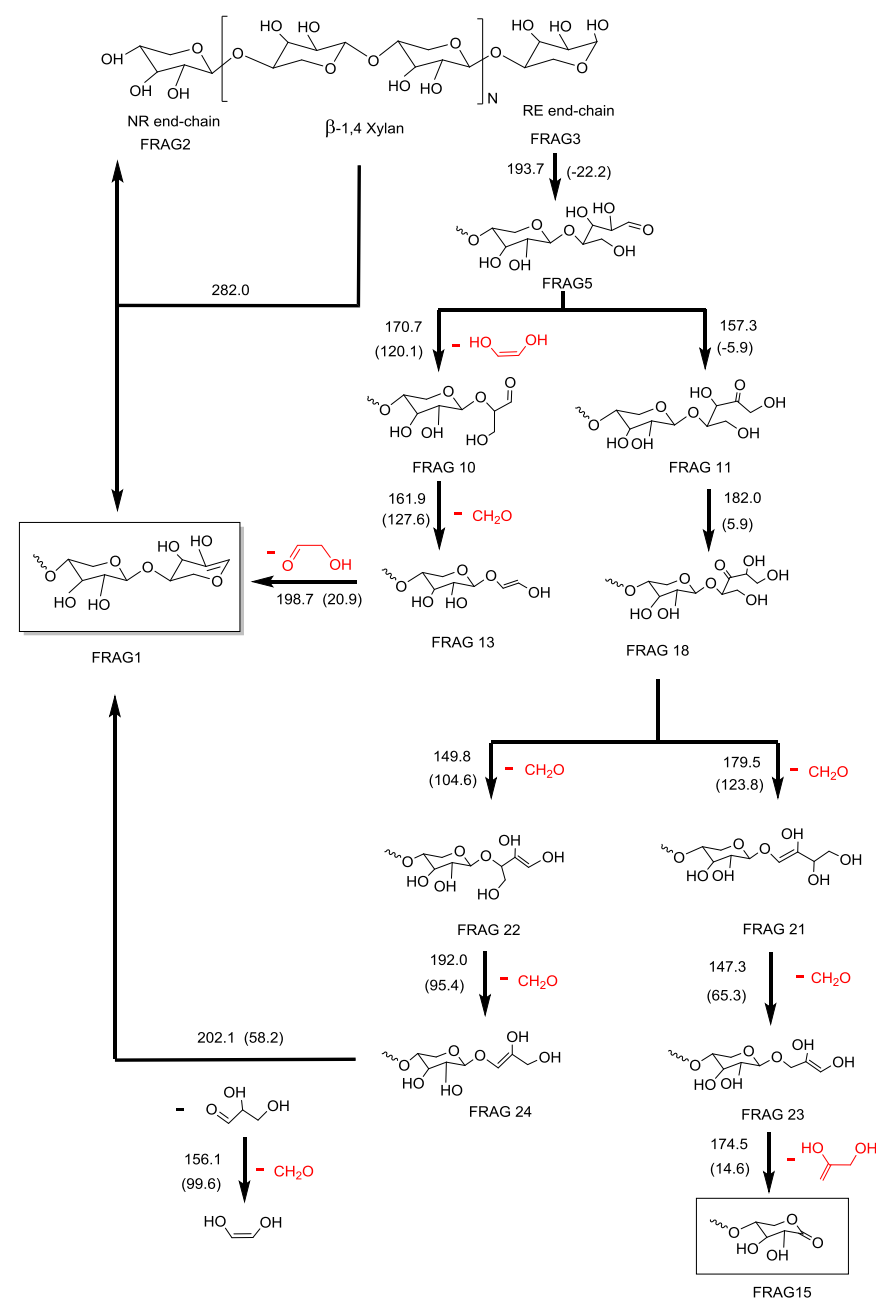

Scheme 26. Simplified scheme of the thermal decomposition of $\beta-1,4-x y l a n$ from unimolecular concerted reactions. Barrier heights at $0 \mathrm{~K}$ (including ZPE) have been computed at the CBS-QB3 level of theory. Values in parenthesis are reaction enthalpies at $0 \mathrm{~K}$ (in kJ mol-1).

Zhang et al. ${ }^{10}$ performed an experimental study of the pyrolysis of hemicellulose from corn stover. They applied several treatments to the initial hemicellulose to finally elaborate a structure containing $92 \mathrm{~mol} \%$ of xylose and $6 \mathrm{~mol} \%$ of arabinose in mineral-free conditions. At $500^{\circ} \mathrm{C}$ (773K), acetaldehyde, formaldehyde, hydroxyaldehyde (glycoaldehyde), methylglyoxal, hydroxyacetone (acetol) and carbon monoxide are important light products observed, in good agreement with the species predicted by Schemes 26 and 27 . They also detected 2 -furaldehyde (furfural), dianhydroxylopyranose (DAXP), $\mathrm{CO}_{2}$ and water in significant concentration. As previously discussed, it seems difficult to form furfural from unimolecular concerted reactions. Indeed, the formation of furfural requires successive dehydrations, with excessively high energy barriers, to compete with reactions such as retro-aldol or retro-ene reactions. Likewise, in our mechanism the DAXP is formed by fission of a glycosidic bond in fragment 1 (TS 77 in Scheme 23). However, the competing retro Diels-Alder reaction is more favorable, with a barrier height $89.1 \mathrm{~kJ} \mathrm{~mol}^{-1}$ lower. Other reactions such as keto-enol tautomerizations also involve high energy barriers. For example, ethene-diol or 2-hydroxyprop-2-enal appear in the mechanism but their isomerization into hydroxyaldehyde and methylglyoxal, respectively, requires activation energies close to $250 \mathrm{~kJ} \mathrm{~mol}^{-1}$. 


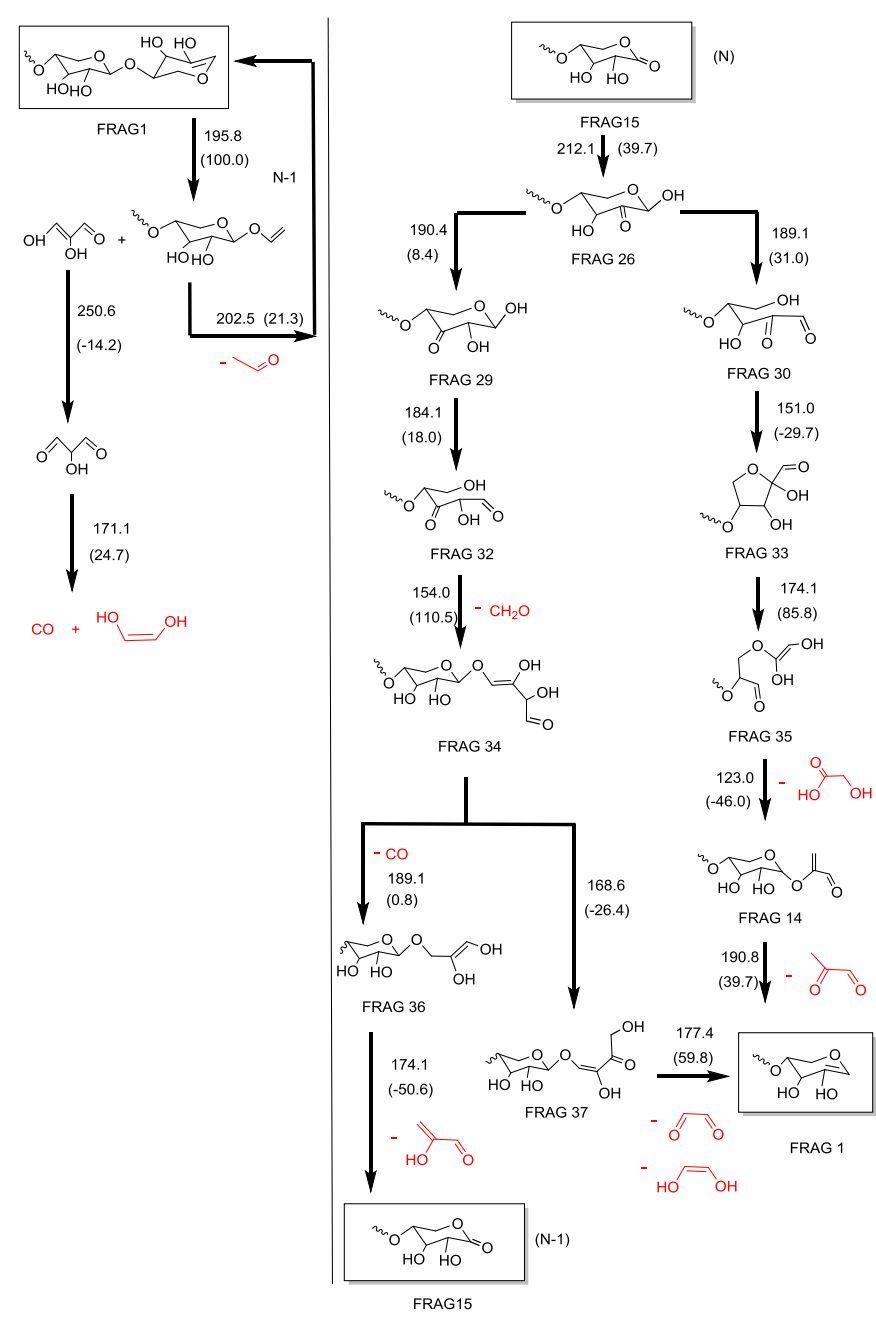

Scheme 27. Simplified scheme of the thermal decomposition of the two key species, namely fragment 1 and fragment 15 . Barrier heights at $0 \mathrm{~K}$ (including ZPE) have been computed at the CBS-QB3 level of theory. Values in parenthesis are reaction enthalpies at $0 \mathrm{~K}$ (in $\mathrm{kJ} \mathrm{mol}-1$ ).

These results can be explained by highly constrained transition states involved into 4-centered reactions. Assisted reactions by water ${ }^{26,33,34}$ or more generally by $\mathrm{R}-\mathrm{OH}^{25}$ could decrease activation energy and promote such reactions ${ }^{35}$. Even if such mechanisms are out of the scope of this study, exploratory calculations performed on the glycosidic bond fissions of $\beta-1,4$-xylan assisted by water, showed that activation energies are lower by around $71 \mathrm{~kJ} \mathrm{~mol}^{-1}$. Even under these conditions, the ring opening of the reductive end-chain remains predominant.

\section{Conclusions}

Electronic calculations performed at the CBS-QB3 level of theory along with canonical transition state theory were used to theoretically investigate the pyrolysis of $\beta-1,4-x y l a n$. This polymer is a suitable model to represent the backbone of many hemicelluloses. A conformation analysis has permitted to define the lowest energy model molecule, with respect to the orientation of the hydroxyl groups. In this structure, all the OHs are oriented towards the ring oxygen. From this low energy conformer, we investigated the thermal decomposition of $\beta-1,4-x y l a n$, considering unimolecular concerted reactions. At each step, we computed the rate coefficients for temperatures ranging from 500 to $1200 \mathrm{~K}$ and we only retained the fragments corresponding to the low energy pathways before to take the next step. Mid-chain and end-chain reactions have been distinguished. Activation energies involved in the mid-chain reactions are significant $\left(E_{a}>280 \mathrm{~kJ}^{-1} \mathrm{~mol}^{-1}\right.$ ), especially for dehydrations. The lower energy path is a glycosidic bond fission leading to an unsaturated chain end (fragment 1 ) while the other fragment corresponds to the reductive end-chain of $\beta-1,4$-xylan (fragment 3 ). The pyrolysis of fragment 1 can be depicted by a closed sequence reaction involving, successively, a retro Diels-Alder and a retro-ene reaction and leading to a new polymer structure similar to fragment 1 , but containing one less monomer. The non-reductive end-chain reacts similarly to mid-chains, i.e. mainly by glycosidic bond fissions involving similar energy barriers. On the other hand, reductive end-chain can easily decompose by ring opening (fragment 5). A detailed analysis of the pathways involved in the decomposition of fragment 5 as well as the successive structures obtained, allowed to isolated two key compounds: the fragment 1 already encountered in the mid-chain decomposition and a lactone structure (fragment 15). The pyrolysis of fragment 15 involves many decomposition 
steps with the formation of new fragments, which finally lead to structures similar to those of fragments 1 and 15 (with one monomer less). Thus, a second closed sequence reaction can be highlighted involving the fragment 15 . These closed sequences can be compared to chain-propagating reactions, even if chain-carriers are polymer fragments rather than radicals. All the energy barriers computed for reductive end-chain reactions are lower than $213 \mathrm{~kJ}^{-\mathrm{mol}^{-1}}$, with many of them between 167 and $188 \mathrm{~kJ} \mathrm{~mol}^{-1}$. Therefore, reductive chain ends reactions are more likely to occur than those involving mid-chains, despite the high degree of polymerization in $\beta-1,4-x y l a n$, which increases the number of glycosidic bonds. The formation of light species such as aldehydes or $\mathrm{CO}$ is consistent with the main pathways proposed in this work. Based on our mechanism, significant concentrations of furfural or dianhydroxylopyranose, often observed in experimental studies of the pyrolysis of xylan, cannot be explained from unimolecular concerted reactions. Indeed, these reactions require dehydration steps but the formation of water involves high energy barriers and is always in competition with an easier reaction pathway. We checked that radical reactions cannot compete with concerted reactions but another explanation could be found in the work of Seshadri and Westmoreland ${ }^{26}$. Assisted $\mathrm{R}-\mathrm{OH}$ reactions could lower energy barriers of 4-centered reactions. However, entropic effects and R-OH concentration must be taken into account to correctly model the role of such assisted reactions. Future work will investigate the kinetic influence of assisted reactions for 4-centered concerted reactions (dehydrations, keto-enol tautomerizations,...) during the thermal decomposition of 1,4- $\beta$-xylan.

\section{Conflicts of interest}

There are no conflicts to declare.

\section{Acknowledgements}

High performance computing resources were partially provided by the EXPLOR center hosted by the University of Lorraine. This work was also granted access to the HPC resources of IDRIS under the allocation 2018-A0010807249 made by GENCI.

\section{Notes and references}

1. Y. Kim, A. E. Thomas, D. J. Robichaud, K. lisa, P. C. St. John, B. D. Etz, G. M. Fioroni, A. Dutta, R. L. McCormick, C. Mukarakate and S. Kim, J. Hazard. Mater., 2020, 400, 123198.

2. A. Gonzalez-Quiroga, K. M. Van Geem and G. B. Marin, Biomass Conversion and Biorefinery, 2017, 7, 305-317.

3. X. Zhou, W. Li, R. Mabon and L. J. Broadbelt, Energy Technology, 2017, 5, 52-79.

4. M. Carrier, R. Fournet, B. Sirjean, S. Amsbury, Y. Benitez-Alfonso, P.-Y. Pontalier and A. V. Bridgwater, Energy Fuels, 2020.

5. $\quad$ S. Wang, B. Ru, H. Lin and Z. Luo, Bioresour. Technol., 2013, 143, 378-383.

6. J. Huang, C. He, L. Wu and H. Tong, Chem. Phys. Lett., 2016, 658, 114-124.

7. J. Huang, C. Liu, H. Tong, W. Li and D. Wu, Computational and Theoretical Chemistry, 2012, 1001, 44-50.

8. Z. Li, C. Liu, X. Xu and Q. Li, Computational and Theoretical Chemistry, 2017, 1117, 130-140.

9. X. Zhou, W. Li, R. Mabon and L. J. Broadbelt, Energy \& Environmental Science, 2018, 11, 1240-1260.

10. J. Zhang, Y. S. Choi, C. G. Yoo, T. H. Kim, R. C. Brown and B. H. Shanks, ACS Sustainable Chemistry \& Engineering, $2015,3,293-301$.

11. X. Yang, Y. Zhao, W. Li, R. Li and Y. Wu, Energy Fuels, 2019, 33, 4352-4360.

12. B. Hu, Q. Lu, Z.-X. Zhang, Y.-T. Wu, K. Li, C.-Q. Dong and Y.-P. Yang, Combust. Flame, 2019, 206, 177-188.

13. Z. Yu, P. Murria, M. W. Easton, J. C. Degenstein, H. Zhu, L. Xu, R. Agrawal, W. N. Delgass, F. H. Ribeiro and H. I. Kenttämaa, J. Phys. Chem. A, 2019, 123, 9149-9157.

14. R. D. Bach, M. N. Glukhovtsev, C. Gonzalez, M. Marquez, C. M. Estévez, A. G. Baboul and H. B. Schlegel, J. Phys. Chem. A, 1997, 101, 6092-6100.

15. V. Guner, K. S. Khuong, A. G. Leach, P. S. Lee, M. D. Bartberger and K. N. Houk, J. Phys. Chem. A, 2003, 107, 11445-11459.

16. M. J. Frisch, G. W. Trucks, H. B. Schlegel, G. E. Scuseria, M. A. Robb, J. R. Cheeseman, G. Scalmani, V. Barone, B. Mennucci, G. A. Petersson and et al., Gaussian 09, Revision D.01, Wallingford CT, 2009.

17. B. Sirjean, R. Fournet, P.-A. Glaude and M. F. Ruiz-López, Chem. Phys. Lett., 2007, 435, 152-156.

18. T. J. Lee and P. R. Taylor, A diagnostic for determining the quality of single-reference electron correlation methods, 1989.

19. C. Eckart, Physical Review, 1930, 35, 1303-1309.

20. J. Lizardo-Huerta, B. Sirjean, R. Bounaceur and R. Fournet, PCCP, 2016, 18, 12231-12251.

21. I. Pena, S. Mata, A. Martin, C. Cabezas, A. M. Daly and J. L. Alonso, PCCP, 2013, 15, 18243-18248.

22. F. Zielinski, S. T. Mutter, C. Johannessen, E. W. Blanch and P. L. A. Popelier, PCCP, 2015, 17, 21799-21809.

23. B. A. Franca and C. O. da Silva, PCCP, 2014, 16, 13096-13102.

24. M. Carrier, R. Fournet, B. Sirjean, S. Amsbury, Y. B. Alfonso, P.-Y. Pontalier and A. Bridgwater, Energy Fuels, 2020, 34, 1423214248.

25. H. B. Mayes and L. J. Broadbelt, J. Phys. Chem. A, 2012, 116, 7098-7106. 
26. V. Seshadri and P. R. Westmoreland, Catal. Today, 2016, 269, 110-121.

27. X. Zhang, J. Li, W. Yang and W. Blasiak, Energy Fuels, 2011, 25, 3739-3746.

28. NIST, http://kinetics.nist.gov.

29. Q. Lu, B. Hu, Z.-x. Zhang, Y.-t. Wu, M.-s. Cui, D.-j. Liu, C.-q. Dong and Y.-p. Yang, Combust. Flame, 2018, 198, $267-277$.

30. K. Werner, L. Pommer and M. Broström, Journal of Analytical and Applied Pyrolysis, 2014, 110, 130-137.

31. U. Räisänen, I. Pitkänen, H. Halttunen and M. Hurtta, Journal of Thermal Analysis and Calorimetry, 2003, 72, 481-488.

32. D. K. Shen, S. Gu and A. V. Bridgwater, Carbohydr. Polym., 2010, 82, 39-45.

33. A. Yamaguchi, N. Muramatsu, N. Mimura, M. Shirai and O. Sato, PCCP, 2017, 19, 2714-2722.

34. M. Wang, C. Liu, Q. Li and X. Xu, Journal of Molecular Modeling, 2015, 21, 296.

35. V. Maliekkal, S. Maduskar, D. J. Saxon, M. Nasiri, T. M. Reineke, M. Neurock and P. Dauenhauer, ACS Catalysis, 2019, 9, 19431955. 